\title{
BASIC SEQUENCES AND SUBSPACES IN LORENTZ SEQUENCE SPACES WITHOUT LOCAL CONVEXITY
}

\author{
BY \\ NICOLAE POPA ${ }^{1}$
}

\begin{abstract}
After some preliminary results (\$1), we give in $\$ 2$ another proof of the result of $N$. J. Kalton [5] concerning the unicity of the unconditional bases of $l_{p}$, $0<p<1$.

Using this result we prove in $\$ 3$ the unicity of certain bounded symmetric block bases of the subspaces of the Lorentz sequence spaces $d(w, p), 0<p<1$. In 84 we show that every infinite dimensional subspace of $d(w, p)$ contains a subspace linearly homeomorphic to $l_{p}, 0<p<1$.

Unlike the case $p>1$ there are subspaces of $d(w, p), 0<p<1$, which contain no complemented subspaces of $d(w, p)$ linearly homeomorphic to $l_{p}$. In fact there are spaces $d(w, p), 0<p<1$, which contain no complemented subspaces linearly homeomorphic to $l_{p}$. We conjecture that this is true for every $d(w, p), 0<p<1$. The answer to the previous question seems to be important: for example we can prove that a positive complemented sublattice $E$ of $d(w, p), 0<p<1$, with a symmetric basis is linearly homeomorphic either to $l_{p}$ or to $d(w, p)$; consequently, a positive answer to this question implies that $E$ is linearly homeomorphic to $d(w, p)$. In $\$ 5$ we are able to characterise the sublattices of $d(w, p), p=k^{-1}$ (however under a supplementary restriction concerning the sequence $\left.\left(w_{n}\right)_{n-1}^{\infty}\right)$, which are positive and contractive complemented, as being the order ideals of $d(w, p)$.

Finally, in $\$ 6$, we characterise the Mackey completion of $d(w, p)$ also in the case $p=k^{-1}, k \in \mathbf{N}$.
\end{abstract}

1. Preliminary results. Let $X$ be a real linear space and $0<p<1$. A function, denoted by \|\| , defined on $X$ with the values in $\mathbf{R}_{+}$, is called a $p$-norm (or briefly a norm) if the following conditions are verified.

1. $\|x\|=0$ if and only if $x=0$.

2. $\|\alpha x\|=|\alpha|^{p}\|x\|$ for $x \in X$ and $\alpha \in \mathbf{R}$.

3. $\|x+y\| \leqslant\|x\|+\|y\|$ for $x, y \in X$.

Then the subsets $U_{n}=\left\{x \in X:\|x\| \leqslant n^{-1}\right\}$, for $n \in \mathbf{N}$, constitute a fundamental system of neighbourhoods of zero for a metric linear topology of $X$. If $X$ is complete with respect to this topology we say that $X$ is a $p$-Banach space.

A sequence $\left(x_{n}\right)_{n=1}^{\infty}$ in $X$ is called a basis if for every $x \in X$ there is a unique sequence of scalars $\left(a_{n}\right)_{n=1}^{\infty}$ such that $x=\sum_{i=1}^{\infty} a_{i} x_{i}$.

The following lemma is essentially known (see Theorem III.6.1, Theorem 6.5 of [10]):

LEMMA 1.1. Let $\left(x_{i}\right)_{i=1}^{\infty}$ be a sequence in $X$. The following assertions are equivalent:

1. The series $\sum_{n=1}^{\infty} x_{\pi(n)}$ converges for every permutation $\pi$ of the integers.

Received by the editors September 13, 1979 and, in revised form, December 4, 1979.

AMS (MOS) subject classifications (1970). Primary 46A45, 46A10; Secondary 46A35.

Key words and phrases. $p$-Banach spaces, symmetric bases, complemented subspaces.

'Supported by Alexander von Humboldt Foundation. 
2. The series $\sum_{i \in A} x_{i}$ converges for every subset $A \subset \mathbf{N}$.

3. For every $\varepsilon>0$ there exists an integer $n$ such that $\left\|\sum_{i \in H} x_{i}\right\| \leqslant \varepsilon$ for every finite set of integers $H$ which satisfies $\min \{i \in H\}>n$.

4. For any bounded sequence of real numbers $\left(a_{n}\right)_{n=1}^{\infty}$, the series $\sum_{i=1}^{\infty} a_{i} x_{i}$ converges, when $\sum_{i=1}^{\infty} x_{i}$ converges.

The proof will be omitted.

A basis $\left(x_{n}\right)_{n=1}^{\infty}$ in $X$ is said to be unconditional if for every $X \ni x=\sum_{i=1}^{\infty} a_{i} x_{i}$, the sequence $\left(a_{n} x_{n}\right)_{n=1}^{\infty}$ verifies one of the equivalent assertions of Lemma 1.1.

If $0<\inf _{n}\left\|x_{n}\right\| \leqslant \sup _{n}\left\|x_{n}\right\|<+\infty$, we say that the basis $\left(x_{n}\right)_{n=1}^{\infty}$ is bounded.

The following corollary is also known.

Corollary 1.2. Let $\left(x_{i}\right)_{i=1}^{\infty}$ be an unconditional bounded basis of $X$. Then

$$
\|x\|=\sup _{\left|b_{i}\right|<1}\left\|\sum_{i=1}^{\infty} a_{i} b_{i} x_{i}\right\|<+\infty .
$$

We have also

LEMMA 1.3. The space $X$ with the p-norm ||| || is a p-Banach space.

Proof. Let $\left(x^{k}\right)_{k=1}^{\infty}$ be a Cauchy sequence in $(X,|||| \mid)$ and let $\varepsilon>0$. Then there exists the sequence of integers $\left(n_{k}\right)_{k=1}^{\infty}$ so that $\left\|x^{n}-x^{n_{1}}\right\| \mid<\varepsilon$ for $n \geqslant n_{1}$ and $\left\|x^{n_{k}}-x^{n_{k+1}}\right\| \mid<\varepsilon / 2^{k}$ for every $k \in N$. Since $(X,\|\|)$ is a $p$-Banach space and $\|x\| \leqslant\|x\|$, there exists $x=\sum_{n=1}^{\infty}\left(x^{n_{k+1}}-x^{n_{k}}\right)+x^{n_{1}} \in X$ and $\left\|x-x^{n}\right\| \leqslant 2 \varepsilon$ for $n \geqslant n_{1}$.

COROLlary 1.4. There exists a constant $0<M<+\infty$ such that

$$
\left\|\sum_{i=1}^{\infty} a_{i} b_{i} x_{i}\right\| \leqslant M\left\|\sum_{i=1}^{\infty} a_{i} x_{i}\right\| \sup _{i \in \mathrm{N}}\left|b_{i}\right|^{p}
$$

where $\left(x_{i}\right)_{i=1}^{\infty}$ is an unconditional bounded basis of $X$ and $x=\sum_{i=1}^{\infty} a_{i} x_{i} \in X$.

Proof. It follows by Lemma 1.3 and by the open mapping theorem.

Two bases $\left(x_{n}\right)_{n=1}^{\infty}$ and $\left(y_{n}\right)_{n=1}^{\infty}$ of $X$ are equivalent and we write $\left(x_{n}\right) \sim\left(y_{n}\right)$, if for every sequence of scalars $\left(a_{n}\right)_{n=1}^{\infty}, \sum_{n=1}^{\infty} a_{n} x_{n}$ converges if and only if $\sum_{n=1}^{\infty} a_{n} y_{n}$ converges. A basis $\left(x_{n}\right)_{n=1}^{\infty}$ of $X$ is called symmetric if every permutation $\left(x_{\pi(n)}\right)_{n=1}^{\infty}$ of $\left(x_{n}\right)_{n=1}^{\infty}$ is a basis of $X$ equivalent to $\left(x_{n}\right)_{n=1}^{\infty}$.

Let $w=\left(w_{i}\right)_{i=1}^{\infty} \in c_{0} \backslash l_{1}$, where $1=w_{1} \geqslant w_{2} \geqslant \cdots \geqslant w_{n}>\cdots \geqslant 0$. For a fixed $p$ with $0<p<1$, let

$$
d(w, p)=\left\{a=\left(a_{i}\right)_{i=1}^{\infty} \in c_{0}:\|a\|_{p, w}=\sup _{\pi} \sum_{i=1}^{\infty}\left|a_{\pi(i)}\right|^{p} \cdot w_{i}<+\infty\right\}
$$

where $\pi$ is an arbitrary permutation of the integers.

Then $X=\left(d(w, p),\|\|_{p, w}\right)$ is a $p$-Banach space and the canonical basis $\left(x_{n}\right)_{n=1}^{\infty}$, is a symmetric basis of $X$ (see [4]). If $p \geqslant 1$ we can define analogously $d(w, p)$, which is a Banach space under the norm

$$
\|a\|_{p, w}=\sup _{\pi}\left(\sum_{i=1}^{\infty}\left|a_{\pi(i)}\right|^{p} \cdot w_{i}\right)^{1 / p}, \quad a=\left(a_{i}\right)_{i=1}^{\infty} \in d(w, p) .
$$


A sequence $\left(y_{n}\right)_{n=1}^{\infty}$ in a $p$-Banach space with a basis $\left(x_{n}\right)_{n=1}^{\infty}$ is called a block basic sequence of $\left(x_{n}\right)_{n=1}^{\infty}$, if there is an increasing sequence of integers $\left(p_{n}\right)_{n=1}^{\infty}$ such that $y_{n}=\sum_{i=p_{n}+1}^{p_{n+1}} a_{i} x_{i}$ with $\left(a_{n}\right)_{n=1}^{\infty}$ scalars.

It is known that $\left(y_{n}\right)_{n=1}^{\infty}$ is a basis of $\overline{\operatorname{Sp}}\left\{y_{n}: n \in \mathbf{N}\right\}$ (see II.5.6 of [10]).

If $E$ and $F$ are two Banach spaces and $0<r<+\infty$, we say that the linear bounded operator $T: E \rightarrow F$ is $r$-absolutely summing if, for any finite set of elements $\left(x_{i}\right)_{i=1}^{n}$ of $E$, there is $c>0$ such that

$$
\left(\sum_{i=1}^{n}\left\|T x_{i}\right\|^{r}\right)^{1 / r} \leqslant c \cdot \sup \left\{\left(\sum_{i=1}^{n}\left|x^{\prime}\left(x_{i}\right)\right|^{r}\right)^{1 / r}: x^{\prime} \in E^{\prime},\left\|x^{\prime}\right\| \leqslant 1\right\} .
$$

Denote the set of all $r$-absolutely summing operators from $E$ to $F$ by $P_{r}(E, F)$.

If $T \in P_{r}(E, F)$ and $r \geqslant 1$ then $\pi_{r}(T)=\inf \{c>0: c$ verifies (1.3) $\}$ is a norm on $P_{r}(E, F)$, which is a Banach space with respect to this norm.

We recall here two more theorems which we need: by

If $A$ is an infinite matrix of real numbers $\left(a_{i j}\right)_{i, j=1}^{\infty}$ and $0<p \leqslant 1$, then we denote

$$
\|A\|_{\infty, p}=\sup _{\|x\|_{\infty}<1}\left(\sum_{j}\left|\sum_{k} a_{j k} x_{k}\right|^{p}\right)^{1 / p}
$$

where $\|x\|_{\infty}=\sup _{k \in N}\left|x_{k}\right|$.

Theorem 1.5 (SEe TheOREM 2 of [2]). Let $0<p<1$. For every matrix $A$ such that $\|A\|_{\infty, p}<\infty$, we have the inequality

$$
\inf \left\{\|d\|_{p /(1-p)} \cdot\|B\|_{\infty, 1}: A=(\operatorname{diag} d) \circ B\right\}<K\|A\|_{\infty, p}
$$

where

$$
d=\left(d_{n}\right)_{n=1}^{\infty} \in l_{p /(1-p)}, \quad\|d\|_{p /(1-p)}=\left(\sum_{i}\left|d_{i}\right|^{p /(1-p)}\right)^{(1-p) / p},
$$

$\operatorname{diag} d$ is the matrix which has on the diagonal the numbers $d_{n}$, and $K$ is a positive constant depending only on $p$.

TheOREM 1.6 (SEE THEOREM 94 of [9]). Let $(X, \mu)$ be a measure space and $H a$ Hilbert space. Then any linear bounded operator from $L^{1}(X, \mu)$ on $H$ is p-absolutely summing for all $0<p<\infty$.

(In fact Maurey proved a stronger version of Theorem 1.6.)

2. The unicity of the unconditional bases of $l_{p}, 0<p<1$. In this section we give a new proof of Kalton's result [5] concerning the unicity of unconditional bases of $l_{p}$, $0<p<1$. The proof follows the idea of Lindenstrauss and Pekzyński's proof concerning the unicity of unconditional bases of $l_{1}$ [7].

TheOREM 2.1. Any two unconditional bounded bases of $l_{p}, 0<p<1$, are equivalent.

Proof. If $\left(e_{n}\right)_{n=1}^{\infty}$ is the canonical basis of $l_{p}, 0<p<1$, let $\left(x_{n}\right)_{n=1}^{\infty}$, where $x_{i}=\sum_{j=1}^{\infty} b_{i j} e_{j}$ such that $\sum_{j=1}^{\infty}\left|b_{i j}\right|^{p}=1$ for every $i \in \mathbf{N}$, another unconditional 
(assumed normalized) basis and let $y=\sum_{i=1}^{\infty} a_{i} x_{i}$ an element of $l_{p}, 0<p<1$. For any $x \in l_{p}$ we denote by $\|x\|_{p}=\left\|\sum_{i=1}^{\infty} \alpha_{i} e_{i}\right\|_{p}=\sum_{i=1}^{\infty}\left|\alpha_{i}\right|^{p}$, the norm of $l_{p}$ for $0<p \leqslant 1$.

Let $A: c_{0} \rightarrow l_{p}$ be the operator defined by the infinite matrix $A=\left(a_{i} b_{i j}\right)_{i, j=1}^{\infty}$. By (1.2) it follows that $A$ is a continuous linear operator, and moreover

$$
\|A\|_{\infty, p}=\sup _{\left|\lambda_{i}\right|<1}\left\|\sum_{i=1}^{\infty} \lambda_{i} a_{i} x_{i}\right\|_{p}^{1 / p} \leqslant M\left\|\sum_{i=1}^{\infty} a_{i} x_{i}\right\|_{p}^{1 / p} .
$$

Theorem 1.5 shows us that for every $\varepsilon>0$ there are $K=K(p)>0$, the diagonal matrix $D=\left(d_{i} \partial_{i j}\right)_{i, j=1}^{\infty}$, where $d=\left(d_{i}\right)_{i=1}^{\infty} \in l_{p /(1-p)}$ and the matrix $C=\left(c_{i j}\right)_{i, j=1}^{\infty}$ such that

$$
\left\{\begin{array}{l}
A=D \cdot C, \\
\|C\|_{\infty, 1}=\sup _{\left|\lambda_{i}\right|<1}\left\|\sum_{j=1}^{\infty}\left(\sum_{i=1}^{\infty} \lambda_{i} c_{i j}\right) e_{j}\right\|_{1}<+\infty, \\
\|d\|_{p /(1-p)} \cdot\|C\|_{\infty, 1} \leqslant K\|A\|_{\infty, p}+\varepsilon .
\end{array}\right.
$$

Theorem 2.b.7 of [8] says that each linear bounded operator $T: c_{0} \rightarrow l_{1}$ is 2-absolutely summing, consequently

$$
\pi_{2}(C) \leqslant K_{G}\|C\|_{\infty, 1}
$$

where $C$ is the operator defined by the matrix $C$ and $K_{G}$ is a universal constant.

Note now that by Hölder's inequality we have

$$
\|D\|=\sup _{\|b\|_{1}<1}\left(\sum_{i=1}^{\infty}\left|d_{i} b_{i}\right|^{p}\right)^{1 / p} \leqslant\|d\|_{p /(1-p)}
$$

where $D: l_{1} \rightarrow l_{p}$ is defined by the matrix $D$ and $b=\left(b_{i}\right)_{i=1}^{\infty} \in l_{1}$. Hence

$$
\begin{aligned}
\left(\sum_{i=1}^{\infty}\left|a_{i}\right|^{2}\right)^{1 / 2} & =\left(\sum_{i=1}^{\infty}\left\|a_{i} x_{i}\right\|_{p}^{2 / p}\right)^{1 / 2} \\
& =(\text { by }(2.2))=\left(\sum_{i=1}^{\infty}\left\|D C\left(e_{i}\right)\right\|_{p}^{2 / p}\right)^{1 / 2} \\
& \leqslant\|D\|\left(\sum_{i=1}^{\infty}\left\|C e_{i}\right\|^{2}\right)^{1 / 2} \leqslant(\text { by }(2.3) \text { and }(2.4)) \\
& \leqslant\|d\|_{p /(1-p)} \cdot K_{G}\|C\|_{\infty, 1} \cdot \sup _{\sum_{i=1}^{\infty}\left|\alpha_{i}\right|<1}\left(\sum_{i=1}^{\infty}\left|\alpha_{i}\right|^{2}\right)^{1 / 2} \leqslant(\text { by }(2.2)) \\
& \leqslant K_{G}\left(K\|A\|_{\infty, p}+\varepsilon\right) \leqslant(\text { by }(2.1)) \\
& \leqslant K K_{G} M\left\|\sum_{i=1}^{\infty} a_{i} x_{i}\right\|_{p}^{1 / p}+\varepsilon K_{G} .
\end{aligned}
$$

Since $\varepsilon$ is arbitrarily small it follows that, for every $y=\sum_{i=1}^{\infty} a_{i} x_{i} \in l_{p}$,

$$
\left(\sum_{i=1}^{\infty}\left|a_{i}\right|^{2}\right)^{1 / 2} \leqslant M K K_{G}\left\|\sum_{i=1}^{\infty} a_{i} x_{i}\right\|_{p}^{1 / p} .
$$


Consequently the operator $U: l_{p} \rightarrow l_{2}$ defined by $U\left(x_{i}\right)=e_{i}, i=1,2, \ldots$, is continuous and verifies

$$
\|U\|_{p, 2}=\sup \left\{\left\|\sum_{i=1}^{\infty} a_{i} e_{i}\right\|: a=\sum_{i=1}^{\infty} a_{i} x_{i},\|a\|_{p} \leqslant 1\right\} \leqslant K K_{G} M .
$$

Denoting by $S_{q}$ the unit ball of $l_{q}, 0<q<\infty$, and by $\bar{\Gamma}^{(1)}\left(S_{p}\right)$ the closure for the topology of $l_{1}$ of the convex and balanced hull of $S_{p}$, it is easy to see that $S_{1} \subseteq \bar{\Gamma}^{(1)}\left(S_{p}\right)$. Then, by (2.6), it follows that

$$
\begin{aligned}
U\left(l_{p} \cap S_{1}\right) & \subset U\left(\bar{\Gamma}^{(1)}\left(S_{p}\right) \cap l_{p}\right) \\
& \subseteq\left(K K_{G} M\right)^{-1} \bar{\Gamma}^{(1)}\left(S_{2}\right) \subset\left(K_{G} K M\right)^{-1} S_{2},
\end{aligned}
$$

and this relation implies that $U$ can be extended to an operator $V: l_{1} \rightarrow l_{2}$ such that we have

$$
\|V\| \leqslant K_{G} K M \text {. }
$$

But Theorem 2.b.6 of [8] says that each linear bounded operator $V: l_{1} \rightarrow l_{2}$ is a 1 -absolutely summing operator and $\pi_{1}(V) \leqslant K_{G}\|V\| \leqslant K_{G}^{2} K M$.

Applying Theorem 1.6 it follows that $V \in P_{p}\left(l_{1}, l_{2}\right)$, hence there is a positive constant $M_{1}$ depending only on $p$ such that

(2.8) implies that

$$
\pi_{p}(V) \leqslant K_{G}^{2} K M M_{1}=K_{1} .
$$

$$
\begin{aligned}
\left(\sum_{i=1}^{\infty}\left|a_{i}\right|^{p}\right)^{1 / p} & =\left(\sum_{i=1}^{\infty}\left\|V\left(a_{i} x_{i}\right)\right\|^{p}\right)^{1 / p} \\
& \leqslant K_{1} \sup _{\left|\lambda_{j}\right|<1}\left(\sum_{i=1}^{\infty}\left|a_{i}\right|^{p}\left|\sum_{j=1}^{\infty} \lambda_{j} b_{i j}\right|^{p}\right)^{1 / p} .
\end{aligned}
$$

On the other hand, denoting by $C^{\prime}$ the adjoint of the operator $C$, we have

$$
\begin{aligned}
\sup _{\left|\lambda_{j}\right|<1}\left(\sum_{i=1}^{\infty}\left|a_{i}\right|^{p} \mid\right. & \left.\left.\sum_{j=1}^{\infty} \lambda_{j} b_{i j}\right|^{p}\right)^{1 / p}=(\text { by }(2.2)) \\
& =\sup _{\left|\lambda_{j}\right|<1}\left(\sum_{i=1}^{\infty}\left|d_{i}\right|^{p}\left|\sum_{j=1}^{\infty} \lambda_{j} c_{i j}\right|^{p}\right)^{1 / p}<\text { (by Hölder's inequality) } \\
& \leqslant \sup _{\left|\lambda_{j}\right|<1}\left(\sum_{i=l}^{\infty}\left|\sum_{j=1}^{\infty} \lambda_{j} c_{i j}\right|\right) \cdot\|d\|_{p /(1-p)} \\
& =\|d\|_{p /(1-p)} \cdot\left\|C^{\prime}\right\|_{\infty, 1} \leqslant(\text { by }(2.2))<K\|A\|_{\infty, p}+\varepsilon \\
& \leqslant(\text { by }(2.1)) \leqslant K M\left\|\sum_{i=1}^{\infty} a_{i} x_{i}\right\|_{p}^{1 / p}+\varepsilon
\end{aligned}
$$

(2.9) and (2.10) imply that, for every $y=\sum_{i=1}^{\infty} a_{i} x_{i} \in l_{p}$,

$$
\left(\sum_{i=1}^{\infty}\left|a_{i}\right|^{p}\right)^{1 / p} \leqslant K_{1} K M\left\|\sum_{i=1}^{\infty} a_{i} x_{i}\right\|_{p}^{1 / p} \leqslant K_{1} K M\left(\sum_{i=1}^{\infty}\left|a_{i}\right|^{p}\right)^{1 / p} .
$$

Thus $\left(x_{i}\right)_{i} \sim\left(e_{i}\right)_{i}$. 
3. Symmetric basic sequences in $d(w, p), 0<p<1$. In this section we prove the analogues of Theorem 3 and Lemma 1 [1] for $0<p<1$. We show moreover the unicity of the symmetric bases in $d(w, p)$ in the case that this space is not included in $l_{1}$. Finally we state some open problems concerning the symmetric basic sequences in $d(w, p), 0<p<1$.

In the proofs of the following two results the techniques of Altshuler, Cassaza and Lin [1] work almost unchanged.

LEMMA 3.1. Let $\left(x_{n}\right)_{n=1}^{\infty}$ be the canonical basis in $d(w, p), 0<p<1$. If $y_{n}=$ $\sum_{i=p_{n}+1}^{p_{n+1}} a_{i} x_{i}, n=1,2, \ldots$, is a bounded block basic sequence of $\left(x_{n}\right)_{n=1}^{\infty}$ such that $\lim _{n} a_{n}=0$, then there is a subsequence of $\left(y_{n}\right)_{n=1}^{\infty}$ which is equivalent to the canonical basis of $l_{p}$.

Proof. Since every change of signs and every permutation of the integers induces an isometry in $d(w, p)$ we may assume, by switching to a subsequence if necessary, that $\left(a_{i}\right)_{i=1}^{\infty}$ is a nonincreasing sequence of positive numbers. Moreover we may assume that $\left\|y_{n}\right\|_{p, w}=1$ for $n \in \mathbf{N}$.

Now let $0<\varepsilon<2^{2}\left(2^{p}-1\right)^{1 / p}\left(2^{p+1}-1\right)^{-1 / p}$. Using the facts that $\lim _{n} a_{n}=0$ and $\left\|y_{n}\right\|_{p, w}=1$ for every $n \in \mathbf{N}$, it is easy to construct by induction two increasing sequences of integers $\left(n_{j}\right)_{j=1}^{\infty}$ and $\left(r_{j}\right)_{j=1}^{\infty}$ such that

$$
\left\{\begin{array}{l}
p_{n_{j}}<r_{j}<p_{n_{j}+1}, \\
Q_{0}=0 \\
Q_{j}=\sum_{k=1}^{j}\left(p_{n_{k}+1}-p_{n_{k}}\right) \leqslant r_{j+1}-p_{n_{j+1}}, \quad j=1,2, \ldots \\
\left(\sum_{i=p_{n_{j}+1}}^{r_{j}}\left(a_{i}\right)^{p} \cdot w_{i-p_{n_{j}}}\right)^{1 / p}<\varepsilon / 2^{j+1}
\end{array}\right.
$$

For the sequence of scalars $\left(\lambda_{j}\right)_{j=1}^{\infty}$ we have

$$
\begin{aligned}
\left\|\sum_{j=1}^{\infty} \lambda_{j} y_{n_{j}}\right\|_{p, w}= & \left\|\sum_{j=1}^{\infty} \lambda_{j}\left[\left(\sum_{i=p_{n_{j}+1}}^{r_{j}} a_{i} x_{i}\right)+\left(\sum_{i=r_{j}+1}^{p_{n_{j}+1}} a_{i} x_{i}\right)\right]\right\|_{p, w} \\
\geqslant & \left\|\sum_{j=1}^{\infty} \lambda_{j}\left(\sum_{i=r_{j}+1}^{p_{n_{j}+1}} a_{i} x_{i}\right)\right\|_{p, w}-\left\|\sum_{j=1}^{\infty} \lambda_{j}\left(\sum_{i=p_{n}+1}^{r_{j}} a_{i} x_{i}\right)\right\|_{p, w} \\
\geqslant & \left\|\sum_{j=1}^{\infty} \lambda_{j}\left(\sum_{i=r_{j}+1}^{p_{n+1}} a_{i} x_{i}\right)\right\|-\sum_{p, w}^{\infty}\left|\lambda_{j}\right|^{p}\left\|\sum_{i=p_{n}+1}^{r_{j}} a_{i} x_{i}\right\|_{p, w} \\
\geqslant & \left(\operatorname{since}\left\{r_{k}-p_{n_{k}}+1, \ldots, p_{n_{k}+1}-p_{n_{k}}\right\}\right. \\
& \cap\left\{r_{j}-p_{n_{j}}+1, \ldots, p_{n_{j}+1}-p_{n_{j}}\right\}=\varnothing \text { and } \\
& \left.\left\{r_{k}+1, \ldots, p_{n_{k}+1}\right\} \cap\left\{r_{j}+1, \ldots, p_{n_{j}+1}\right\}=\varnothing \text { for } k \neq j\right)
\end{aligned}
$$




$$
\begin{aligned}
& \geqslant \sum_{j=1}^{\infty}\left|\lambda_{j}\right|^{p}\left(\sum_{i=r_{j}+1}^{p_{n_{j}+1}}\left(a_{i}\right)^{p} \cdot w_{Q_{j-1}-r_{j}+i}\right)-\sum_{j=1}^{\infty}\left(\varepsilon / 2^{j+1}\right)^{p}\left|\lambda_{j}\right|^{p} \\
& \geqslant \sum_{j=1}^{\infty}\left|\lambda_{j}\right|^{p}\left(\sum_{i=r_{j}+1}^{p_{n_{j}+1}}\left(a_{i}\right)^{p} \cdot w_{i-p_{n_{j}}}\right)-\left[\varepsilon^{p} 2^{-p}\left(2^{p}-1\right)^{-1}\right] \cdot \sup _{j}\left|\lambda_{j}\right|^{p} \\
& \geqslant \sum_{j=1}^{\infty}\left|\lambda_{j}\right|^{p}\left[1-\varepsilon^{p} 2^{-p(j+1)}-\varepsilon^{p} 2^{-p}\left(2^{p}-1\right)^{-1}\right] \\
& \geqslant\left[1-\frac{\varepsilon^{p}\left(2^{p+1}-1\right)}{2^{2 p}\left(2^{p}-1\right)}\right] \sum_{j=1}^{\infty}\left|\lambda_{j}\right|^{p} .
\end{aligned}
$$

On the other hand we have

$$
\left\|\sum_{j=1}^{\infty} \lambda_{j} y_{n_{j}}\right\|_{p, w} \leqslant \sum_{j=1}^{\infty}\left|\lambda_{j}\right|^{p}\left\|y_{n_{j}}\right\|_{p, w}=\sum_{j=1}^{\infty}\left|\lambda_{j}\right|^{p} .
$$

We shall use forward a special block basic sequence. Let $\left(x_{n}\right)_{n=1}^{\infty}$ be a symmetric basis in the $p$-Banach space $X$. If $0 \neq a=\sum_{n=1}^{\infty} a_{n} x_{n} \in X$ and if $\left(p_{i}\right)_{i=1}^{\infty}$ is an increasing sequence of integers, let $y_{n}^{(a)}=\sum_{i=p_{n}+1}^{p_{n+1}} a_{i-p_{n}} x_{i}, n \in \mathrm{N}$. Then $\left(y_{n}^{(a)}\right)_{n=1}^{\infty}$ is a bounded block basic sequence of $\left(x_{n}\right)_{n=1}^{\infty}$, and we shall call it a block of type I of $\left(x_{n}\right)_{n=1}^{\infty}$.

THEOREM 3.2. Every bounded block basic sequence of $\left(x_{n}\right)_{n=1}^{\infty}$ in $d(w, p), 0<p<$ 1 , has a subsequence equivalent either to the unit vector basis of $l_{p}$ or to a block basic sequence of type I of $\left(x_{n}\right)_{n=1}^{\infty}$.

Proof. Let $y_{n}=\sum_{i=p_{n}+1}^{p_{n+1}} a_{i} x_{i}, n=1,2, \ldots$ We may assume that $\left\|y_{n}\right\|_{p, w}=1$ and that $a_{p_{n}+1} \geqslant \cdots \geqslant a_{p_{n+1}} \geqslant 0$ for every $n \in \mathbf{N}$. If $\sup _{n}\left(p_{n+1}-p_{n}\right)<+\infty$, then it is clear that $\left(y_{n}\right)_{n} \sim\left(x_{n}\right)_{n}$, hence $\left(y_{n}\right)_{n=1}^{\infty}$ is equivalent to a block basic sequence of type I of $\left(x_{n}\right)_{n=1}^{\infty}$. Assume now that $\sup _{n}\left(p_{n+1}-p_{n}\right)=\infty$. Let $b_{i}=\sup _{n}\left|a_{p_{n}+i}\right|$ for $i \in \mathbf{N}$. It is easy to prove that $\lim _{i} b_{i}=0$.

Case I. Assume that for every $\varepsilon>0$ there exists $m$ such that $\left\|\sum_{i=p_{n}+m}^{p_{n+1}+m} a_{i} x_{i}\right\|_{p, w}<$ $\varepsilon$ for every $n$ so that $p_{n+1}-p_{n} \geqslant m$. Since $\sup _{n}\left(p_{n+1}-p_{n}\right)=+\infty$, we may assume that $p_{n+2}-p_{n+1} \geqslant p_{n+1}-p_{n}$ for every $n \in \mathbf{N}$. Define now $z_{n}=\sum_{i=1}^{p_{n+1}-p_{n}} a_{i+p_{n}} x_{i}$, $n \in \mathbf{N}$. Then $\left\|z_{n}\right\|_{p, w}=\left\|y_{n}\right\|_{p, w}=1$ for $n \in \mathbf{N}$. By hypothesis and using the fact that there is a subsequence $\left(n_{k}\right)_{k=1}^{\infty}$ such that the sequences $\left(a_{i+p_{n_{k}}}\right)_{k=1}^{\infty}$ converge simultaneously for $i \leqslant m$, we can find a Cauchy subsequence of $\left(z_{n}\right)_{n=1}^{\infty}$. Hence we may assume that $\lim _{n} z_{n}=z=\sum_{i=1}^{\infty} c_{i} x_{i} \in d(w, p)$. It is clear that $z \neq 0$.

Since $\left(y_{n}\right)_{n=1}^{\infty}$ is a bounded block basic sequence of $\left(x_{n}\right)_{n=1}^{\infty}$ it is well known that $K=\sup _{n}\left\|P_{n}\right\|<+\infty$, where $P_{n}\left(\sum_{i=1}^{\infty} a_{i} y_{i}\right)=\sum_{i=1}^{n} a_{i} y_{i}$ (see III.2.11 of [10]). Consequently we can find a subsequence $\left(z_{n_{i}}\right)_{i=1}^{\infty}$ such that $\sum_{i=1}^{\infty}\left\|z_{n_{i}}-z\right\|_{p, w}<1 / 2 K$. Define now

$$
u_{i}=\sum_{k=p_{n_{i}}+1}^{p_{n_{i}+1}} c_{k-p_{n_{i}}} x_{k}, \quad i=1,2, \ldots
$$


Then $\left(u_{i}\right)_{i=1}^{\infty}$ is a block basic sequence of type I of $\left(x_{n}\right)_{n-1}^{\infty}$ and $\sum_{i=1}^{\infty}\left\|y_{n_{i}}-u_{i}\right\|_{p, w}<$ $\sum_{i=1}^{\infty}\left\|z_{n_{i}}-z\right\|_{p, w}<1 / 2 K$, hence by the Krein-Milman-Rutman Theorem (see Theorem III.2.13 of [10]) it follows that $\left(y_{n_{i}}\right)_{i=1} \sim\left(u_{i}\right)_{i=1}$.

Case II. There exists an $\varepsilon>0$ such that for every $m \in \mathbf{N}$ there exists $n(m)$ such that $p_{n+1}-p_{n} \geqslant m$ and $\left\|\sum_{i=p_{n}+m}^{p_{n+1}+m} a_{i} x_{i}\right\|_{p, w}>\varepsilon$. Then there exists an increasing sequence of integers $\left(n_{i}\right)_{i=1}^{\infty}$ such that

$$
p_{n_{i}+1}-p_{n_{i}}>i \text { and }\left\|\sum_{j=p_{n_{i}+i}}^{p_{n_{i}+1}} a_{j} x_{j}\right\|_{p, w}>\varepsilon \quad \text { for some } i \in \mathbf{N} \text {. }
$$

Since $\lim _{i} b_{i}=0$ we may assume that $\left(a_{j}\right)_{j=1}^{\infty}$ is a decreasing sequence. Let

$$
z_{i}=\sum_{j=p_{m}+1}^{p_{m+1}} a_{j} x_{j} \quad \text { for } i \in \mathbf{N} .
$$

Then $\varepsilon \leqslant\left\|z_{i}\right\|_{p, w} \leqslant\left\|y_{i}\right\|_{p, w}=1$ for $i \in \mathbf{N}$, also $\left(z_{i}\right)_{i=1}^{\infty}$ is a bounded block basic sequence of $\left(x_{n}\right)_{n=1}^{\infty}$ and the coefficients of $z_{i}$ converge to zero. By Lemma 3.1 it follows that there exists a subsequence $\left(t_{i}\right)_{i=1}^{\infty}$ of $\left(z_{i}\right)_{i=1}^{\infty}$ which is equivalent to the canonical basis $\left(e_{i}\right)_{i=1}^{\infty}$ of $l_{p}$. Since $\left(y_{n_{i}}\right)_{i=1}^{\infty}$ dominates $\left(t_{i}\right)_{i=1}^{\infty}$ (i.e. $\left\|\sum_{i=1}^{\infty} b_{i} y_{n_{i}}\right\|_{p, w}$ $<+\infty$ implies that $\left\|\sum_{i=1}^{\infty} b_{i} t_{i}\right\|_{p, w}<\infty$ for every sequence $\left.\left(b_{i}\right)_{i=1}^{\infty}\right)$ then $\left\|\sum_{i=1}^{\infty} d_{i} y_{n_{i}}\right\|_{p, w}<\infty$ implies that $\sum_{i=1}^{\infty}\left|d_{i}\right|^{p}<\infty$ for every sequence of scalars $\left(d_{i}\right)_{i=1}^{\infty}$. On the other hand, since $\left\|y_{n_{i}}\right\|_{p, w}=1$ for every $i \in \mathbf{N}, \sum_{i=1}^{\infty}\left|d_{i}\right|^{p}<\infty$ implies that $\left\|\sum_{i=1}^{\infty} d_{i} y_{n_{i}}\right\|_{p, w}<\infty$, hence $\left(y_{n_{i}}\right)_{i} \sim\left(e_{i}\right)_{i}$.

In the remainder of this section we study the unicity of the symmetric bases of the subspaces of $d(w, p), 0<p<1$. We shall of ten use the following notion. Let $X$ be a $p$-Banach space with a separating dual $X$ (this is the case whenever $X$ has a basis). We consider on $X$ the finest locally convex topology weaker than the original one i.e. the Mackey topology on $X$. It is easy to see that the Mackey topology on $X$ is generated by the neighbourhoods $((1 / n) \operatorname{co}(S))_{n=1}^{\infty}$, where $S=$ $\{x \in X:\|x\|<1\}$. Then the completion of $X$ in the Mackey topology, $\tilde{X}$, is a Banach space. It is interesting that $\overline{d(w, p)}, 0<p<1$, may be exactly $l_{1}$. The routine proof of the following proposition will be omitted.

Proposition 3.3. A bounded set $A \subset d(w, p), 0<p<\infty$, is precompact if and only if for every $\varepsilon>0$ there is $n \in \mathbf{N}$ such that

$$
\sup _{\pi} \sum_{i=n}^{\infty}\left|a_{\pi(i)}\right|^{p} \cdot w_{i}<\varepsilon
$$

uniformly for $a=\left(a_{i}\right)_{i=1}^{\infty} \in A$. (The supremum is taken over all permutations of integers.)

Proposition 3.4. Let $0<p<1$. Then $\overline{d(w, p)}=l_{1}$ if and only if $d(w, p) \subset l_{1}$. Moreover if $d(w, p) \nsubseteq l_{1}$ then $d(w, p) \approx l_{1}$ (i.e. $d(w, p)$ is not linearly homeomorphic to $l_{1}$ ).

Proof. If $d(w, p) \nsubseteq l_{1}$, let $a=\left(a_{i}\right)_{i=1}^{\infty} \in d(w, p) \backslash l_{1}, A=\left\{x_{i}: i \in \mathbf{N}\right\}$ and $f \in$ $d(w, p)^{\prime}$. We shall show that

$$
\lim _{i} f\left(x_{i}\right)=0 .
$$


Indeed if (3.3) is not true, there exist the sequence $\left(i_{k}\right)_{k=1}^{\infty}$ of integers and $\alpha>0$ such that $\left|f\left(x_{i_{k}}\right)\right| \geqslant \alpha$ for every $k \in \mathbf{N}$. We consider $b=\left(b_{i}\right)_{i=1}^{\infty}$, where

$$
b_{i}= \begin{cases}a_{j} \operatorname{sign} f\left(x_{i_{j}}\right), & i=i_{j}, j \in \mathbf{N}, \\ 0, & i \neq i_{j}, j \in \mathbf{N} .\end{cases}
$$

It is clear that $b \in d(w, p) \backslash l_{1}$. But $\infty=\alpha . \sum_{i=1}^{\infty}\left|a_{i}\right|<\left|\sum_{j=1}^{\infty} f\left(x_{j}\right) b_{j}\right|<\infty$, which is contradictory. Then (3.3) is true and $A$ is weakly relatively compact. On the other hand (3.2) is not verified for $A$ and $p=1$, hence $A$ is not a relatively compact subset of $d(w, 1)$. Since the canonical mapping $i: d(w, p) \rightarrow d(w, 1), 0<p<1$, is clearly continuous, it is obvious that on $d(w, p)$ the topology induced by $\overline{d(w, p)}$ is stronger than that induced by $d(w, 1)$, consequently $A$ is not a relatively compact subset of $\overparen{d(w, p)}$.

If $\widetilde{d(w, p)} \approx l_{1}$, then, $A$ being weakly relatively compact in $\widetilde{d(w, p)}$ (since clearly $d(w, p)^{\prime}=\widetilde{\left.d(w, p)^{\prime}\right)}$ it is also relatively compact in $\overline{d(w, p)}$, which is a contradiction. Thus $\widetilde{d(w, p)} \approx l_{1}$. Conversely, if $d(w, p) \subset l_{1}$, we denote by $I: d(w, p) \rightarrow l_{1}$ the canonical mapping. We shall show that $I$ is continuous, that is there exists $K>0$ such that, for every $a \in d(w, p)$, we have

$$
\sum_{i=1}^{\infty}\left|a_{i}\right| \leqslant K\|a\|_{p, w}^{1 / p}
$$

It is clear that it suffices to prove (3.4) only for positive decreasing sequences $\left(a_{i}\right)_{i=1}^{\infty} \in d(w, p)$. If (3.4) is not true, then for every $n \in \mathbf{N}$ there is the positive decreasing sequence $a^{(n)}=\left(a_{i}^{(n)}\right)_{i=1}^{\infty} \in d(w, p)$, such that

$$
2^{n}\left\|a^{(n)}\right\|_{p, w}^{1 / p}<\sum_{i=1}^{\infty} a_{i}^{(n)}
$$

Denote by $b^{(n)}=2^{-n}\left\|a^{(n)}\right\|_{p, w}^{-1 / p} a^{(n)}$. Then $\left\|\sum_{n=1}^{\infty} b^{(n)}\right\|_{p, w}<\sum_{n=1}^{\infty}\left\|b^{(n)}\right\|_{p, w}<$ $\sum_{n=1}^{\infty} 1 / 2^{n p}<\infty$, consequently it follows that $b=\sum_{n=1}^{\infty} b^{(n)} \in l_{1}$. But (3.5) implies that $1<\sum_{i=1}^{\infty} b_{i}^{(n)}$ for every $n \in \mathbf{N}$, hence $\left\|\sum_{n=1}^{\infty} b^{(n)}\right\|_{1}=\sum_{n=1}^{\infty} \sum_{i=1}^{\infty} b_{i}^{(n)}=+\infty$, which is a contradiction. Hence $I$ is continuous. On the other hand the canonical mapping $J: l_{p} \rightarrow d(w, p)$ is continuous and consequently the extensions $\tilde{I}: \tilde{l}_{p} \rightarrow$ $\widetilde{d(w, p)}$ and $\tilde{J}: \widetilde{d(w, p)} \rightarrow l_{1}$ are continuous. Since clearly $\tilde{l}_{p}=l_{1}$ for $0<p<1$, and $\tilde{J} \circ \tilde{I}=\mathrm{id}_{l_{1}}$, it follows that $d(w, p)=l_{1}$.

We shall give an example of space $d(w, p), 0<p<1$, for which $d(w, p) \subset l_{1}$. (It is known [4] that $d(w, p) \leftarrow l_{1}$ for $p \geqslant 1$.)

EXAMPLE 3.5. Let $w_{n}=(1+|\log n|)^{-1}$ for $n>1$ and $0<p<1$. Then $d(w, p) \subset$ $l_{1}$.

Proof. Our proof is indirect. We denote by

$$
M(t)= \begin{cases}\frac{t^{p}}{1+|\log t|}, & t \in(0,1], \\ 0, & t=0 .\end{cases}
$$

Then $M(t)$ is a continuous nondecreasing function on $[0,1]$, with $\sup _{t \in(0,1]} M(2 t) / M(t)<\infty$, that is an Orlicz function (see [5]). We consider now 
the locally convex Orlicz sequence space $l_{M}=\left\{a=\left(a_{i}\right)_{i=1}^{\infty}:\|a\|_{M}=\sum_{i=1}^{\infty} M\left(\left|a_{i}\right|\right)\right.$ $<+\alpha\}$. We shall show that

$$
l_{M}=d(w, p)
$$

It is clear that $l_{M}=l_{M_{p}}$, and that $d(w, 1)=l_{M_{1}}$ implies that $d(w, p)=l_{M_{p}}$, where

$$
M_{q}(t)= \begin{cases}\frac{t^{q}}{1+\left|\log t^{q}\right|}, & t \in(0,1], \\ 0, & t=0, \quad 0<q<+\infty .\end{cases}
$$

Consequently it is sufficient to show that

$$
d(w, 1)=l_{M_{1}}=\left\{a=\left(a_{i}\right)_{i=1}^{\infty}: \sum_{i=1}^{\infty} \frac{\left|a_{i}\right|}{1+|\log | a_{i}||}<+\infty\right\} .
$$

Theorem 4.e. 2 of [8] says that $d(w, 1)=l_{M_{1}}$ if and only if there exists $\gamma>0$ such that

$$
\sum_{n=1}^{\infty} 1 / W^{-1}\left(\gamma w_{n}\right)<+\infty
$$

where $W(x)=(1+\log x)^{-1}$ for $x \geqslant 1$.

In our case $W^{-1}\left(\gamma w_{n}\right)=e^{1 /(\gamma-1)} n^{1 / \gamma}$, hence, for every $0<\gamma<1,(3.7)$ and also (3.6) are true. But Theorem 3.3 of [5] shows us that $\widetilde{d(w, p)}=l_{\hat{M}}$, where $\hat{M}$ is the largest Orlicz convex function on $[0,1]$ such that $\hat{M}(x) \leqslant M(x), x \in[0,1]$. Since $K(p) t \leqslant t^{p} /(1+|\log t|) \leqslant t^{p}$ for $t \in(0,1]$, where $K(p)>0$ depends only on $p$, it follows that $\hat{M}$ is equivalent to the function $N(t)=t$, hence

$$
\widetilde{d(w, p)}=l_{\hat{M}}=l_{1} \text {. }
$$

By Proposition 3.4 it follows that $d(w, p) \subset l_{1}$.

REMARK 3.6.1. Since $M(t)$ is not equivalent to $N(t)=t^{p}$ (i.e. there are not the relations $\left.0<\inf _{t \in(0,1]} M(t) / \mathbf{N}(t) \leqslant \sup _{t \in(0,1]} M(t) / \mathrm{N}(t)<+\infty\right)$ the previous space $d(w, p)$ is a $p$-Banach space, other than $l_{p}$, whose dual is $l_{\infty}$.

2. This space is moreover an example of a $p$-Banach space $X$ with a unique unconditional basis, other than $l_{p}$, for which $\tilde{X}$ has a unique unconditional basis. Indeed $\lim _{x \rightarrow 0} M(x) / x=+\infty$, hence by Theorem 7.6 of $[5], d(w, p)=l_{M}$ has a unique unconditional basis.

There are spaces $d(w, p), 0<p<1$, for which $d(w, p) \notin l_{1}$.

Proposition 3.7. Let $\left(w_{i}\right)_{i=1}^{\infty} \in c_{0} \backslash l_{1}$ be a decreasing sequence of positive numbers such that there exist an increasing sequence of integers $\left(n_{j}\right)_{j=1}^{\infty}$, the scalars $b>0$ and $0 \leqslant \gamma<p /(1-p)($ where $0<p<1)$ such that

$$
\begin{gathered}
n_{j+1}-n_{j} \leqslant n_{j+2}-n_{j+1}, \quad j \in \mathbf{N} . \\
n_{j+1}-n_{j} \leqslant b j^{\gamma}, \quad j \in \mathbf{N} . \\
w_{n} \leqslant 1 / j \quad \text { if } n \geqslant n_{j}, j \in \mathbf{N} .
\end{gathered}
$$

Then $d(w, p) \notin l_{1}$. 
Proof. Let

$$
a_{i}= \begin{cases}1 & \text { if } i \leqslant n_{1}, \\ j^{-\beta / p}\left(n_{j+1}-n_{j}\right)^{-1 / p} & \text { if } n_{j}<i<n_{j+1}\end{cases}
$$

where

$$
0<\beta \leqslant p-(1-p) \gamma .
$$

By (3.9) it follows that $\left(a_{i}\right)_{i=1}^{\infty}$ is decreasing to zero and (3.11) implies that

$$
\begin{aligned}
\|a\|_{p, w} & =\sum_{i=1}^{n_{1}} w_{i}+\sum_{j=1}^{\infty} \sum_{i=n_{j}+1}^{n_{j+1}} \frac{w_{i}}{j^{\beta}\left(n_{j+1}-n_{j}\right)} \\
& \leqslant \sum_{i=1}^{n_{1}} w_{i}+\sum_{j=1}^{\infty} \frac{1}{j^{1+\beta}}<+\infty .
\end{aligned}
$$

On the other hand

$$
\begin{aligned}
\|a\|_{1} & =\sum_{i=1}^{n_{j}} 1+\sum_{j=1}^{\infty} \sum_{i=n_{j}+1}^{n_{j+1}} \frac{1}{j^{\beta / p}\left(n_{j+1}-n_{j}\right)^{1 / p}} \\
& =n_{1}+\sum_{j=1}^{\infty} \frac{1}{j^{\beta / p}\left(n_{j+1}-n_{j}\right)^{(1 / p)-1}} \\
& \geqslant\left(\text { by (3.10)) } \geqslant n_{1}+b^{1-1 / p} \sum_{j=1}^{\infty} \frac{1}{j^{\beta / p} \cdot j^{\gamma(1 / p-1)}}\right. \\
& \geqslant\left(\text { by (3.12)) } \geqslant n_{1}+b^{1-1 / p} \sum_{j=1}^{\infty} \frac{1}{j}=\infty,\right.
\end{aligned}
$$

thus $a=\left(a_{i}\right)_{i=1}^{\infty} \notin l_{1}$.

The space $d(w, p), 0<p<1$, with $w_{n}=1 / n$ for $n \geqslant 1$, satisfies the conditions of Proposition 3.7. In Theorem 4 of [1] it is proved that every two symmetric bounded bases of a subspace of $d(w, p), p \geqslant 1$, are equivalent. Similarly we can state the following still open problem.

Problem 1. Let $X \subset d(w, p), 0<p<1$, be a subspace and $\left(y_{n}\right)_{n=1}^{\infty}$ and $\left(z_{n}\right)_{n=1}^{\infty}$ two symmetric bounded bases of $X$. Are $\left(y_{n}\right)_{n=1}^{\infty}$ and $\left(z_{n}\right)_{n=1}^{\infty}$ equivalent?

We are unable to give an answer to Problem 1, however the following theorem is true:

TheOREM 3.8. Let $d(w, p), 0<p<1$, such that $d(w, p) \nsubseteq l_{1}$. Then $d(w, p)$ has a unique bounded symmetric basis.

Proof. Let $\left(y_{n}\right)_{n=1}^{\infty}$ be a bounded symmetric basis of $l_{p}$ other than $\left(x_{n}\right)_{n=1}^{\infty}$. By Proposition 3.4 the hypothesis implies that $\overline{d(w, p)} \approx l_{1}$, hence

$$
\lim _{m} x_{n}^{\prime}\left(y_{m}\right)=0 \text { for every } n \in \mathbf{N},
$$

where $\left(x_{n}^{\prime}\right)_{n=1}^{\infty}$ is the biorthogonal sequence in $d(w, p)^{\prime}$ associated to $\left(x_{n}\right)_{n=1}^{\infty}$ (i.e. $x_{n}^{\prime}\left(x_{m}\right)=\partial_{m n}$ for every $\left.m, n \in N\right)$. Indeed if (3.13) is not true for $n_{0} \in \mathbf{N}$, there exist the subsequence $\left(y_{m_{m}}\right)_{i=1}^{\infty}$ of $\left(y_{n}\right)_{n=1}^{\infty}$ and $\alpha>0$ such that $\alpha<\left|x_{n_{0}}^{\prime}\left(y_{m_{m}}\right)\right|$ for every $i \in \mathbf{N}$. Then for every scalar $\left(a_{i}\right)_{i=1}^{n}$, there are $\varepsilon_{i}= \pm 1, i=1,2, \ldots, n$ and 
$0<M<\infty$, such that

$$
\begin{aligned}
\alpha \sum_{i=1}^{n}\left|a_{i}\right| & \leqslant x_{n_{0}}^{\prime}\left(\sum_{i=1}^{n} a_{i} \varepsilon_{i} y_{m_{i}}\right) \leqslant\left\|\sum_{i=1}^{n} a_{i} \varepsilon_{i} y_{m_{i}}\right\|_{p, w}^{-} \\
& \leqslant \sum_{i=1}^{n}\left|a_{i}\right|\left\|y_{m_{i}}\right\|_{p, w}^{\sim} \leqslant M \sum_{i=1}^{n}\left|a_{i}\right|,
\end{aligned}
$$

where $\|x\|_{p, w}$ is the norm of the element $x \in \widetilde{d(w, p)}$. This inequality shows us that $\widetilde{d(w, p)} \approx l_{1}$, which is a contradiction. (3.13) implies by Proposition 3.1 of [5] that there exists a subsequence $\left(y_{n_{i}}\right)_{i=1}^{\infty}$ of $\left(y_{n}\right)_{n=1}^{\infty}$ which is equivalent to a block basic sequence of $\left(x_{n}\right)_{n=1}^{\infty}$.

The basis $\left(y_{n}\right)_{n=1}^{\infty}$ is a symmetric basis, then $\left(y_{n_{i}}\right)_{i=1}^{\infty} \sim\left(y_{n}\right)_{n=1}^{\infty}$ and consequently we may assume that $y_{m}=\sum_{i=p_{m}+1}^{p_{m+1}} b_{i} x_{i}, m \in \mathbf{N}$. Moreover by Lemma 3.1 it follows that $\lim \sup _{n}\left|b_{n}\right| \neq 0$. (If not then $\left(y_{n}\right)_{n} \sim\left(e_{n}\right)_{n}$.)

Since $\left(x_{n}\right)_{n=1}^{\infty}$ is symmetric, we may also assume that there is $\varepsilon>0$ such that for every $m \in \mathbf{N}$ there is $p_{m}+1 \leqslant k_{m} \leqslant p_{m+1}$ so that $\left|b_{k_{m}}\right|>\varepsilon$. If $\sum_{n=1}^{\infty} a_{n} y_{n}$ converges in $d(w, p)$, then for every $n \in \mathbf{N}$,

$$
\left\|\sum_{i=1}^{n} a_{i} x_{k_{i}}\right\|_{p, w} \leqslant \frac{1}{\varepsilon^{p}}\left\|\sum_{i=1}^{n} b_{k_{i}} a_{i} x_{k_{i}}\right\|_{p, w} \leqslant \frac{1}{\varepsilon^{p}}\left\|\sum_{i=1}^{n} a_{i} y_{i}\right\|_{p, w},
$$

hence $\sum_{n=1}^{\infty} a_{n} x_{n}$ converges in $d(w, p)$. If we interchange the roles of $\left(x_{n}\right)_{n=1}^{\infty}$ and $\left(y_{n}\right)_{n=1}^{\infty}$ we deduce the equivalence of these two bases.

We can state a weaker version of Problem 1.

Problem 1a. Let $d(w, p) \subset l_{1}, 0<p<1$. Is the canonical basis of $d(w, p)$ the unique bounded symmetric basis of $d(w, p)$ ? Finally we have

TheOREM 3.9. Let $X \subset d(w, p), 0<p<1$, be a subspace which has a bounded symmetric basis $\left(y_{n}\right)_{n=1}^{\infty}$ verifying the equality (3.13). Then any other basis $\left(z_{n}\right)_{n=1}^{\infty}$ of $X$, which has the same properties as $\left(y_{n}\right)_{n=1}^{\infty}$ is equivalent to this.

Proof. If $X \approx l_{p}, 0<p<1$, then by Theorem 2.1 it follows that $\left(y_{n}\right)_{n} \sim\left(z_{n}\right)_{n}$. Otherwise, Proposition 3.1 of [5] implies that $\left(y_{n}\right)_{n=1}^{\infty}$ is equivalent to a block basic sequence $\left(u_{n}\right)_{n=1}^{\infty}$ of $\left(x_{n}\right)_{n=1}^{\infty}, u_{n}=\sum_{i=q_{n}+1}^{q_{n+1}} a_{i} x_{i}, n=1,2, \ldots$, and $\left(z_{n}\right)_{n=1}^{\infty}$ is equivalent to a block basic sequence $v_{m}=\sum_{n=r_{m}+1}^{r_{m+1}} b_{n} y_{n}, m=1,2, \ldots$, of $\left(y_{n}\right)_{n}^{\infty}$. Moreover we may assume that $\lim \sup _{n \rightarrow \infty} b_{n} \neq 0$ (otherwise Lemma 3.1 implies that $\left(z_{n}\right)_{n} \sim\left(e_{n}\right)_{n}$ which contradicts our assumption). Reasoning as in Theorem 3.8 we obtain that $\left(z_{n}\right)_{n=1}^{\infty}$ domainates $\left(y_{n}\right)_{n=1}^{\infty}$. By interchanging the roles of $\left(y_{n}\right)_{n=1}^{\infty}$ and $\left(z_{n}\right)_{n=1}^{\infty}$ we deduce the conclusion.

Let us mention the following problem.

Problem 2. Is there a subspace $X \subset d(w, p), 0<p<1$, different from $l_{p}$ and $d(w, p)$, such that $\tilde{X}=l_{1}$ ?

Remark that a negative answer to Problem 2 and a positive one to Problem 1a imply that Theorem 3.9 is true without any restriction concerning the basis $\left(y_{n}\right)_{n=1}^{\infty}$.

4. Complemented subspaces and sublattices of $d(w, p), 0<p<1$. We prove now the version for $0<p<1$ of Theorem 1 of [1]. By $\left(x_{n}\right)_{n=1}^{\infty}$ we mean forward the canonical basis of $d(w, p)$. 
LEMMA 4.1. For every bounded block basic sequence $\left(y_{n}\right)_{n=1}^{\infty}$ of $\left(x_{n}\right)_{n=1}^{\infty}$ there is a block basic sequence of $\left(y_{n}\right)_{n=1}^{\infty}$ which is equivalent to the canonical basis of $l_{p}$.

Proof. Let $y_{n}=\sum_{i=q_{n}+1}^{q_{n+1}} a_{i} x_{i}, n=1,2, \ldots$ Let us remark that, since $\inf _{n}\left\|y_{n}\right\|_{p, w}>0, \sum_{i=1}^{\infty} a_{i} x_{i}$ does not converge in $d(w, p)$. On the other hand, if $\sup _{n}\left\|\sum_{i=1}^{n} a_{i} x_{i}\right\|_{p, w}<\infty$, then $\sum_{i=1}^{\infty} a_{i} x_{i}$ converges in $d(w, p)$. Hence $\sup _{k \leqslant m}\left\|\sum_{i=k}^{n} y_{i}\right\|_{p, w}=+\infty$.

Let $\left(p_{k}\right)_{k=1}^{\infty}$ be an increasing sequence of integers such that

$$
\sup _{n}\left\|\sum_{i=p_{n}+1}^{p_{n+1}} y_{i}\right\|_{p, w}=\infty \text {. }
$$

Also let

$$
z_{n}=\left\|\sum_{i=p_{n}+1}^{p_{n+1}} y_{i}\right\|_{p, w}^{-1 / p} \cdot\left(\sum_{i=p_{n}+1}^{p_{n+1}} y_{i}\right), \quad n \in \mathbf{N} .
$$

Then the block basic sequence $\left(z_{n}\right)_{n=1}^{\infty}$ of $\left(y_{n}\right)_{n=1}^{\infty}$ satisfies the conditions of Lemma 3.1 and, consequently, there is a subsequence $\left(z_{n j}\right)_{j=1}^{\infty}$ of $\left(z_{n}\right)_{n=1}^{\infty}$, which is equivalent to $\left(e_{n}\right)_{n=1}^{\infty}$.

Using Lemma 4.1 we can prove

TheOREM 4.2. Let $X \subset d(w, p), 0<p<1$, be a (closed) subspace of infinite dimension. Then there is a closed subspace $Y \subset X$ such that $Y \approx l_{p}$.

Proof. By Proposition III.2.15 of [10] it follows that $X$ contains a bounded basic sequence $\left(y_{n}\right)_{n=1}^{\infty}$ which is equivalent to a block basic sequence $\left(z_{n}\right)_{n=1}^{\infty}$ of $\left(x_{n}\right)_{n=1}^{\infty}$. Then Lemma 4.1 gives us a subspace of $\overline{\operatorname{Sp}}\left\{z_{n}: n \in \mathbf{N}\right\}$ which is linearly homeomorphic to $l_{p}$. Consequently $X$ contains a subspace $Y \approx l_{p}$.

REMARK 4.3. In Corollary 17 of [3] it is shown that every (closed) subspace of infinite dimension $X$ of $d(w, p), 1 \leqslant p<\infty$, contains a subspace $Y$ complemented in $d(w, p)$ and linearly homeomorphic to $l_{p}$.

This assertion is not true in the case $0<p<1$. Indeed in [12] it is shown that the subspace of $l_{p}, 0<p<1, \quad Y=\overline{\operatorname{Sp}}\left\{u_{n}: n \in \mathrm{N}\right\} \approx l_{p}$, where $u_{n}=$ $n^{-1 / p} \sum_{i=n(n-1) / 2+1}^{n(n+1) / 2} e_{i}, n=1,2, \ldots$, does not contain any infinite dimensional subspace which is complemented in $l_{p}$. Consequently, let $X$ be a subspace of $d(w, p)$ linearly homeomorphic to $l_{p}$ and let $\left(z_{i}\right)_{i=1}^{\infty}$ be a bounded basis of $X$. We consider the block basic sequence $u_{n}=n^{-1 / p} \sum_{j=n(n-1) / 2+1}^{n(n+1) / 2} z_{j}, n=1,2, \ldots$ Then $Y$ $=\overline{\operatorname{Sp}}\left\{u_{n}: n \in \mathbf{N}\right\} \approx l_{p}$ does not contain any complemented infinite dimensional subspace of $d(w, p)$.

Remark 4.3 shows us that there are subspaces linearly homeomorphic to $l_{p}$ (in fact isometric to $l_{p}$ ) which are not complemented in $d(w, p)$ for $0<p<1$. We can prove moreover that there are examples of spaces $d(w, p), 0<p<1$, without any complemented subspace linearly homeomorphic to $l_{p}$.

EXAMPLE 4.4. Let $w_{n}=(1+|\log n|)^{-1}$ for every $n \in \mathbf{N}$ and let $0<p<1$. Then any complemented subspace of $d(w, p)$ with an unconditional basis is linearly homeomorphic to $d(w, p) \approx l_{p}$. 
Proof. By Example 3.5 and Remark 3.6 it follows that $d(w, p) l_{M} \approx l_{p}$, where

$$
M(t)= \begin{cases}\frac{t}{1+|\log t|} & \text { if } t \in(0,1], \\ 0 & \text { if } t=0 .\end{cases}
$$

Moreover, the canonical basis of $d(w, p)$ is the unique unconditional bounded basis of $d(w, p)$. But a theorem of Kalton (see Theorem 7.2 of [5]) shows us that if $l_{F}$ is a nonlocally convex Orlicz sequence space, then any two unconditional bounded bases of $l_{F}$ are equivalent if and only if any complemented subspace with an unconditional basis is isomorphic to $l_{F}$. We conclude applying this result.

Example 4.4 motivates the following question:

Problem 3. Let $d(w, p), 0<p<1$. It is true that there does not exist a complemented subspace of $d(w, p)$ which is linearly homeomorphic to $l_{p}$ ?

First we give some results which seem to indicate an affirmative answer to Problem 3. First we study the positive complemented sublattices of $d(w, p)$, $0<p<1$, which have a symmetric basis. Let $X$ be a $p$-Banach space, $0<p<1$, which is simultaneously a vector lattice verifying the condition

$$
|a|<|b| \text { implies }\|a\| \leqslant\|b\| \text { for every } a, b \in X \text {. }
$$

We call such a space $X$ a $p$-Banach lattice. (Analogously it is defined a Banach lattice.) It is clear that, extending the order relation to $\tilde{X}$ (whenever the last space exists), $X$ is a sublattice of $\tilde{X}$. Let $X$ be a vector lattice and $Y \subset X$ a sublattice of $X$ (i.e. $x \in Y$ implies that $|x| \in Y$ ) which has the property that $x \in X, y \in Y$ and $|x| \leqslant|y|$ imply that $x \in Y$. Then we call $Y$ to be an order ideal of $X$. If $A \subset X$ is a subset of $X$, then we denote by $I_{X}(A)=\{x \in X: \exists a \in A$ and $0<\lambda \in \mathbf{R}$, such that $|x| \leqslant \lambda|a|\}$, the order ideal generated by $A$.

It is clear now that $d(w, p), 0<p<1$, with the canonical order relation is a $p$-Banach lattice and, moreover, an order ideal of the space $\omega$ of all sequences of real numbers. Now we can extend (under certain conditions) Lemma 2.a.11 of [8] to the $p$-Banach lattices.

Lemma 4.5. Let $X$ be a $p$-Banach lattice which is an order ideal of $\omega,\left(\nu_{n}\right)_{n=1}^{\infty} \in c_{0}$ a sequence of positive real numbers, $0 \leqslant y_{n}=\sum_{i \in \sigma_{n}} \alpha_{i} x_{i}$, and $0 \leqslant z_{n}=\sum_{j \in \psi_{n}} \beta_{j} x_{j}$, $n \in \mathbf{N}$, two bounded basic sequences of $X$ (where $x_{n}=\left(\partial_{n i}\right)_{i=1}^{\infty}$ for $n \in \mathbf{N}$ ) such that $\sigma_{n} \cap \psi_{m}=\varnothing$ for every $n, m \in \mathbf{N}$. If there exists a positive and continuous projection $P$ from $X$ onto $\overline{\operatorname{Sp}}\left\{\nu_{n} y_{n}+z_{n}: n \in \mathbf{N}\right\}$, then the sequence $\left(z_{n}\right)_{n}$ dominates $\left(\nu_{n} y_{n}\right)_{n}$.

Proof. Let $P\left(y_{i}\right)=\sum_{j=1}^{\infty} c_{j}^{(i)}\left(\nu_{j} y_{j}+z_{j}\right)$ and $P\left(z_{i}\right)=\sum_{j=1}^{\infty} d_{j}^{(i)}\left(\nu_{j} y_{j}+z_{j}\right), \quad i=$ $1,2, \ldots$, where $c_{j}^{(i)} \geqslant 0$ and $d_{j}^{(i)} \geqslant 0$. Since the basis $\left(x_{n}\right)_{n=1}^{\infty}$ is clearly an unconditional basis of $X$, there is a positive and continuous projection $Q$ such that $Q\left(x_{n}\right)=x_{n}$ for $n \in \sigma_{i}$, where $i \in \mathbf{N}$, and $Q\left(x_{n}\right)=0$ otherwise. Then $Q P\left(z_{i}\right)=$ $\sum_{j=1}^{\infty} d_{j}^{(i)} \nu_{j} y_{j}, i \in \mathbf{N}$, and $Q P$ may be considered as an operator from $\overline{\operatorname{Sp}}\left\{z_{i}: i \in \mathbf{N}\right\}$ to $\overline{\operatorname{Sp}}\left\{y_{n}: n \in \mathbf{N}\right\}$ defined by the infinite matrix with positive entries $\left(d_{j}^{(i)} \nu_{j}\right)_{i, j=1}^{\infty}$. Then the diagonal matrix defines an operator

$$
D: \overline{\operatorname{Sp}}\left\{z_{i}: i \in \mathbf{N}\right\} \rightarrow \bar{I}_{\omega}\left(\overline{\operatorname{Sp}}\left\{y_{i}: i \in \mathbf{N}\right\}\right)=\bar{I}_{X}\left(\overline{\operatorname{Sp}}\left\{y_{i}: i \in \mathbf{N}\right\}\right) .
$$


$D$ is obviously positive and $0 \leqslant D(x) \leqslant Q P(x)$ for every $0 \leqslant x \in \overline{\operatorname{Sp}}\left\{y_{i}: i \in \mathbf{N}\right\}$. Since $X$ is a $p$-Banach lattice we have

$$
\|D x\| \leqslant\|Q P x\| \leqslant M\|x\| \quad \text { for every } 0 \leqslant x \in \overline{\operatorname{Sp}}\left\{y_{i}: i \in \mathbf{N}\right\},
$$

consequently $D$ is a continuous operator.

Assume now that $\sum_{n=1}^{\infty} a_{n} z_{n}$ converges in $\overline{\operatorname{Sp}}\left\{z_{i}: i \in \mathbf{N}\right\}$. Then $D\left(\sum_{n=1}^{\infty} a_{n} z_{n}\right)=$ $\sum_{n=1}^{\infty} a_{n} d_{n}^{(n)} \nu_{n} y_{n}$ converges in $\bar{I}_{X}\left(\overline{\operatorname{Sp}}\left\{y_{i}: i \in \mathbf{N}\right\}\right)$. Since $0<c_{n}^{(n)}\left(\nu_{n} y_{n}+z_{n}\right)<P\left(y_{n}\right)$ for every $n \in \mathbf{N}$, then $\left|c_{n}^{(n)}\right| \leqslant M_{1}$ for $n \in \mathbf{N}$. On the other hand $\nu_{n} c_{n}^{(n)}+d_{n}^{(n)}=1$, $n=1,2, \ldots$, hence $\lim _{n} d_{n}^{(n)}=1$ and, consequently, $\sum_{n=1}^{\infty} a_{n} \nu_{n} y_{n}$ converges.

We can now prove

THEOREM 4.6. Let $X$ be an order ideal of $\omega$, which is a p-Banach lattice and assume that its canonical basis $\left(x_{n}\right)_{n=1}^{\infty}$ is symmetric. If $\left(y_{n}\right)_{n=1}^{\infty}$ is a positive block of type I of $\left(x_{n}\right)_{n=1}^{\infty}$ then $\left(y_{n}\right)_{n} \sim\left(x_{n}\right)_{n}$ if and only if $E=\overline{\operatorname{Sp}}\left\{y_{n}: n \in \mathbf{N}\right\}$ is a positive complemented sublattice of $X$ (i.e. there exists a positive and continuous projection $P$ from $X$ onto $E$ ).

Proof. Let $\sum_{n=1}^{\infty} a_{n} x_{n} \in X, a_{1} \neq 0$ and $y_{n}=\sum_{i=p_{n}+1}^{p_{n+1}} a_{i-p_{n}} x_{i}$ for every $n \in \mathbf{N}$, where $a_{i} \geqslant 0$. We may assume that all $a_{i} \leqslant 1$. Since $\left(x_{n}\right)_{n=1}^{\infty}$ is a symmetric basis of $X$, there exists $M>1$ such that

$$
\frac{1}{M}\left\|\sum_{n} b_{n} x_{p_{n}+1}\right\| \leqslant\left\|\sum_{n=1}^{\infty} b_{n} x_{n}\right\| \leqslant M\left\|\sum_{n=1}^{\infty} b_{n} x_{p_{n}+1}\right\|
$$

for every $\Sigma_{n} b_{n} x_{n} \in X$. Suppose now that $\left(y_{n}\right)_{n} \sim\left(x_{n}\right)_{n}$. Let $K>0$ such that $\left\|\sum_{n=1}^{\infty} b_{n} y_{n}\right\| \leqslant K\left\|\sum_{n=1}^{\infty} b_{n} x_{n}\right\|$ for every $\sum_{n} b_{n} x_{n} \in X$. Define $P\left(\sum_{n=1}^{\infty} b_{n} x_{n}\right)=$ $\sum_{n=1}^{\infty}\left(b_{p_{n}+1} / a_{1}\right) y_{n}$ for $\sum_{n} b_{n} x_{n} \in X$. Since $\left(y_{n}\right)_{n} \sim\left(x_{n}\right)_{n}, P$ is well defined, $0<P$ and $\|P\| \leqslant K a_{1}^{-p}$.

Conversely, let $P: K \rightarrow E$ be a positive and continuous projection. If $x=$ $\Sigma_{n} b_{n} x_{n} \in X$ and $\|x\| \leqslant 1$, we choose the sequence of integers $1=n_{1}<n_{2}<\ldots$ such that $\left\|\sum_{j=n_{i}}^{\infty} b_{j} x_{j}\right\| \leqslant 1 / 2^{i}, i=1,2, \ldots$ For $n_{i} \leqslant m<n_{i+1}, i=1,2, \ldots$, we put

$$
z_{m}= \begin{cases}\sum_{j=1}^{i} a_{j} x_{p_{m}+j} & \text { if } p_{m}+i<p_{m+1} \\ y_{m} & \text { if } p_{m}+i>p_{m+1}\end{cases}
$$

and

$$
w_{m}= \begin{cases}\left(y_{m}-z_{m}\right) /\left\|y_{m}-z_{m}\right\| & \text { if } y_{m} \neq z_{m}, \\ 0 & \text { if } y_{m}=z_{m} .\end{cases}
$$

Let $\nu_{n}=\left\|y_{n}-z_{n}\right\|$ for $n \in \mathbf{N}$. Then $\nu_{m}, w_{m}, z_{m}$ are positive, $y_{m}=\nu_{m} w_{m}+z_{m}$ for $m \in \mathbf{N},\left(\nu_{n}\right)_{n=1}^{\infty} \in c_{0}$ and $\inf \left(z_{n}, w_{m}\right)=0$ for $m, n \in N$. Since $0<a_{i}<1, i=$ $1,2, \ldots$, it follows that 


$$
\begin{aligned}
\left\|\sum_{n=1}^{\infty} b_{n} z_{n}\right\| & =\left\|\sum_{i=1}^{\infty} \sum_{m=n_{i}}^{n_{i+1}-1} b_{m} z_{m}\right\| \\
& \leqslant\left\|\sum_{i=1}^{\infty} \sum_{m=n_{i}}^{n_{i+1}-1} b_{m} \sum_{j=1}^{p_{m+1}-p_{m}} x_{p_{m}+j}\right\| \\
& \leqslant\left(\operatorname{since}\left(x_{i}\right)_{i=1}^{\infty} \text { is a symmetric basis }\right)<M\left\|\sum_{i=1}^{\infty} \sum_{m=n_{i}}^{n_{i+1}-1} b_{m} x_{p_{m}+1}\right\| \\
& \leqslant M \sum_{i=1}^{\infty}\left\|\sum_{j=n_{i}}^{\infty} b_{j} x_{p_{j}+1}\right\| \leqslant M^{2} \sum_{i=1}^{\infty}\left\|\sum_{j=n_{i}}^{\infty} b_{j} x_{j}\right\| \\
& \leqslant M^{2} \sum_{i=1}^{\infty} \frac{1}{2^{i}}<+\infty .
\end{aligned}
$$

Consequently $\sum_{n=1}^{\infty} b_{n} z_{n}$ converges and, applying Lemma $4.5, \sum_{n=1}^{\infty} b_{n} \nu_{n} w_{n}$ converges, therefore $\left\|\sum_{n=1}^{\infty} b_{n} y_{n}\right\|<\infty$. Conversely if $\sum_{n=1}^{\infty} b_{n} y_{n}$ converges, then $\sum_{n=1}^{\infty} a_{1} b_{n} x_{p_{n}+1}$ converges. Since $\left(x_{n}\right)_{n=1}^{\infty}$ is a symmetric basis, then $a_{1}\left(\sum_{n=1}^{\infty} b_{n} x_{n}\right)$ converges. Consequently $\left(y_{n}\right)_{n} \sim\left(x_{n}\right)_{n}$.

Proposition 4.7. Let $X$ be a separable p-Banach lattice with the property that every order interval $[0, x]$, where $X \ni x>0$, is compact. Then there is in $X a$ normalized sequence $\left(x_{n}\right)_{n=1}^{\infty}$ of positive pairwise disjoint (i.e. $\inf \left(x_{i}, x_{j}\right)=x_{i} \wedge x_{j}=$ $0, i \neq j)$ elements such that $\left(x_{n}\right)_{n=1}^{\infty}$ is a basis simultaneously of $X$ and of $\tilde{X}$. Particularly $\left(x_{n}\right)_{n=1}^{\infty}$ is an unconditional basis of $X$. Moreover $X$ is an order ideal of $\tilde{X}$.

Proof. We prove first the second assertion. Let $y \in X$ and $z \in \tilde{X}$ such that $0 \leqslant z \leqslant y$. If $0 \leqslant z_{n} \in X$ and $\lim _{n} z_{n}=z$ in $\tilde{X}$, then, since $\tilde{X}$ is a $p$-Banach lattice, $\lim _{n} z_{n} \wedge y=z \wedge y=z$ in $\tilde{X}$. On the other hand $z_{n} \wedge y \in[0, y] \subset X$ and, by hypothesis, there is a subsequence $\left(z_{n_{k}} \wedge y\right)_{k=1}^{\infty}$ which converges in $X$ (and consequently in $\tilde{X}$ ). Hence $\lim _{n} z_{n_{k}} \wedge y=z \in X$ and $X$ is an order ideal in $\tilde{X}$.

Remark now that the hypothesis implies that $[0, y]$ is compact in $\tilde{X}$ for $0 \leqslant y \in$ $\tilde{X}$. But a Walsh's result (see [13]) asserts that a Banach lattice $X$ such that every order interval $[0, x]$ is compact, has a normalized basis of positive pairwise disjoint elements. Consequently there exists a subsequence $\left(x_{n}\right)_{n=1}^{\infty}$ of $\tilde{X}$ of positive pairwise disjoint elements, with $0<\inf _{n}\left\|x_{n}\right\|^{\sim} \leqslant \sup _{n}\left\|x_{n}\right\|^{\sim}<\infty$ (where $\|x\|^{\sim}$ is the norm of the element $x \in \tilde{X}$ ), such that we have a unique expansion $x=\sum_{n=1}^{\infty} a_{n} x_{n}$ with $0 \leqslant a_{n}$, for every $0 \leqslant x \in \tilde{X}$.

Since $X$ is an order ideal of $\tilde{X}$, there is a subset $A \subseteq N$ such that $x_{i} \in X$ for every $i \in A$. Let $0 \leqslant x \in X$. Since $[0, x]$ is compact in $X$, it follows that $x=$ $\sum_{n \in A} a_{n} x_{n}$, the convergence being with respect to the topology of $X$. Hence $\left(x_{n}\right)_{n \in A}$ is a basis of $X$. It is easy to see that $\left(x_{n}\right)_{n \in A}$ is a bounded basis (see also Proposition 3.2 of [5]). Consequently we may assume that $\left\|x_{n}\right\|=1$ for every $n \in A$. Since $X$ is dense in $\tilde{X}$ and $x_{i} \wedge x_{j}=0$ for $i \neq j$, it follows that $A=\mathbf{N}$. The remaining assertion is a consequence of Lemma 1.1 .

Remark now that in view of Proposition 3.3 it follows that $[0, x]$ is a compact set of $d(w, p), 0<p<1$, for every $0 \leqslant x \in d(w, p)$. Consequently, by Proposition 4.7, 
every sublattice of $d(w, p)$ has a basis of positive pairwise disjoint elements.

We can now state the analogue of Corollary 14 of [3].

THEOREM 4.8. Let $E$ be a positive complemented sublattice of $d(w, p), 0<p<1$, which has a symmetric basis. Then $E$ is linearly homeomorphic either to $d(w, p)$ or to $l_{p}$.

Proof. Let $\left(y_{n}\right)_{n=1}^{\infty}$ be the symmetric basis of $E$ whose elements are all positive and pairwise disjoint. If $y_{n}=\sum_{i=1}^{\infty} t_{n i} x_{i}$ for $n \in \mathbf{N}$, then $\lim _{n} t_{n i}=0$ for every $i \in \mathbf{N}$ and, by Proposition 3.1 of [5], there is a subsequence $\left(y_{n_{k}}\right)_{k=1}^{\infty}$ of $\left(y_{n}\right)_{n=1}^{\infty}$ which is equivalent to a bounded block basic sequence $\left(z_{n}\right)_{n=1}^{\infty}$ of $\left(x_{n}\right)_{n=1}^{\infty}$. Moreover repeating the proof of Proposition 1.a.9 of [8], it follows that we can choose $z_{n} \geqslant 0$ for $n \in \mathbf{N}$ such that $\overline{\mathrm{Sp}}\left\{z_{n}: n \in \mathbf{N}\right\}$ is a positive complemented sublattice of $d(w, p) .\left(y_{n}\right)_{n=1}^{\infty}$ being a symmetric basis it follows that $\left(y_{n}\right)_{n} \sim\left(z_{n}\right)_{n}$. Then we can apply Theorem 3.2 , consequently if $E \approx l_{p}$, there exists a positive block of type I $\left(u_{n}\right)_{n=1}^{\infty}$ of $\left(x_{n}\right)_{n=1}^{\infty}$ such that $\left(y_{n}\right)_{n} \sim\left(u_{n}\right)_{n}$. Using again the proof of Proposition 1.a.9 of [8], we may assume moreover that $\overline{\mathrm{Sp}}\left\{u_{n}: n \in \mathbf{N}\right\}$ is a positive complemented sublattice of $d(w, p)$. By Theorem 4.6, we obtain now that $\left(u_{n}\right)_{n=1}^{\infty}$, also and $\left(y_{n}\right)_{n=1}^{\infty}$, is equivalent to $\left(x_{n}\right)_{n=1}^{\infty}$.

In connection with Theorem 4.8 we can state a weaker version of Problem 3.

Problem 3a. Let $d(w, p), 0<p<1$. Is there a positive complemented sublattice $E \subset d(w, p)$ linearly homeomorphic to $l_{p}$ ?

If we have a negative answer to Problem 3a, then Theorem 4.8 shows us that the positive complemented sublattices with a symmetric basis of $d(w, p), 0<p<1$, are linearly homeomorphic to $d(w, p)$. We gave only a partially (negative) answer to Problem $3 \mathrm{a}$, when we assume, supplementarily, that the positive projection $P$ : $d(w, p) \rightarrow E$ is a contraction, i.e. $\|P\| \leqslant 1$.

5. Positive and contractive complemented sublattices of $d(w, 1 / k), k \in \mathbf{N}$. In this section we shall characterise, under certain conditions, the positive and contractive complemented sublattices of $d(w, 1 / k), k \in \mathbf{N}$. We prove first an inequality:

LEMMA 5.1. Let $k \in \mathbf{N},\left(w_{i}\right)_{i=1}^{\infty} \in c_{0} \backslash l_{1}$ such that $w_{1} \geqslant w_{2} \geqslant \cdots \geqslant 0$ and $\alpha_{1}$ $=\cdots=\alpha_{l}>\alpha_{l+1} \geqslant \alpha_{l+2} \geqslant \cdots \geqslant \alpha_{m}>0$ for some $l<m$. Then denoting by $s_{n}=\sum_{i=1}^{n} w_{i}$, by

$$
C(l)=\left(\begin{array}{l}
1 \\
k
\end{array}\right)\left(\alpha_{l+1}\right)^{1 / k}\left(\alpha_{l}^{1-1 / k}-\alpha_{l+1}^{1-1 / k}\right) w_{l+1} s_{l}^{k-1}
$$

and by

$$
\partial_{l}(n)= \begin{cases}1 & \text { for } n \geqslant l+1 \\ 0 & \text { for } n<l+1\end{cases}
$$

we have the following inequality

$$
\begin{aligned}
A_{n} & =\sum_{i=1}^{n-1} s_{i}^{k}\left(\alpha_{i}-\alpha_{i+1}\right)+s_{n}^{k} \alpha_{n}+\partial_{l}(n) C(l) \\
& \leqslant\left(\sum_{i=1}^{n} \alpha_{i}^{1 / k} \cdot w_{i}\right)^{k}=B_{n} \text { for every } n \leqslant m .
\end{aligned}
$$


Proof. We use the induction for $n$. If $n \leqslant l$ it is nothing to prove. Now let $n=l+1$. Then

$$
\begin{aligned}
A_{l+1}= & \alpha_{l} s_{l}^{k}+\left(s_{l+1}^{k}-s_{l}^{k}\right) \alpha_{l+1}+\left(\begin{array}{l}
1 \\
k
\end{array}\right) \alpha_{l+1}^{1 / k}\left(\alpha_{l}^{1-1 / k}-\alpha_{l+1}^{1-1 / k}\right) w_{l+1} s_{l}^{k-1} \\
= & \alpha_{l} s_{l}^{k}+\alpha_{l+1}\left(\left(\begin{array}{l}
2 \\
k
\end{array}\right) w_{l+1}^{2} s_{l}^{k-2}+\cdots+w_{l+1}^{k}\right)+\left(\begin{array}{l}
1 \\
k
\end{array}\right) \alpha_{l+1}^{1 / k} \alpha_{l}^{1-1 / k} \cdot w_{l+1} s_{l}^{k-1} \\
\leqslant & \left(\text { since } \alpha_{l+1} \leqslant \alpha_{i}, i=1, \ldots, l\right) \\
\leqslant & \alpha_{l} s_{l}^{k}+\left(\begin{array}{l}
1 \\
k
\end{array}\right) \alpha_{l+1}^{1 / k} \alpha_{l}^{1-1 / k} \cdot w_{l+1} s_{l}^{k-1} \\
& +\left(\left(\begin{array}{l}
2 \\
k
\end{array}\right) \alpha_{l+1}^{2 / k} \alpha_{l}^{1-2 / k} \cdot w_{l+1}^{2} s_{l}^{k-2}+\cdots+\alpha_{l+1} w_{l+1}^{k}\right) \\
& \left(\sum_{i=1}^{l+1} \alpha_{i}^{1 / k} \cdot w_{i}\right)^{k}=B_{l+1} .
\end{aligned}
$$

Thus (5.1) is proved in this case.

Assume now that (5.1) is true for $n-1 \geq l+1$. We have

$$
\begin{aligned}
A_{n} & =A_{n-1}+\left(s_{n}^{k}-s_{n-1}^{k}\right) \alpha_{n} \\
& =A_{n-1}+\left(\left(\begin{array}{l}
1 \\
k
\end{array}\right) s_{n-1}^{k-1} \cdot w_{n}+\left(\begin{array}{l}
2 \\
k
\end{array}\right) s_{n-1}^{k-2} \cdot w_{n}^{2}+\cdots+w_{n}^{k}\right) \alpha_{n} \\
& \leqslant\left(\text { since } \alpha_{i} \geqslant \alpha_{i+1} \text { for } i \leqslant n\right) \\
& \leqslant A_{n-1}+\left[\left(\begin{array}{l}
1 \\
k
\end{array}\right)\left(\sum_{i=1}^{n-1} \alpha_{i}^{1 / k} \cdot w_{i}\right)^{k-1} \alpha_{n}^{1 / k} \cdot w_{n}+\cdots+\alpha_{n} w_{n}^{k}\right] \\
& \leqslant(\text { by induction hypothesis }) \\
& <\left(\sum_{i=1}^{n-1} \alpha_{i}^{1 / k} \cdot w_{i}\right)^{k}+\left[\left(\begin{array}{l}
1 \\
k
\end{array}\right)\left(\sum_{i=1}^{n-1} \alpha_{i}^{1 / k} \cdot w_{i}\right)^{k-1} \alpha_{n}^{1 / k} \cdot w_{n}+\cdots+\alpha_{n} w_{n}^{1 / k}\right] \\
& =\left(\sum_{i=1}^{n} \alpha_{i}^{1 / k} \cdot w_{i}\right)^{k}=B_{n} . \square
\end{aligned}
$$

LEMMA 5.2. With the notations of Lemma 5.1, if $\left(\beta_{i}\right)_{i=1}^{m}$ are positive numbers such that

$$
\sum_{i=1}^{n} \beta_{i} \leqslant s_{n}^{k} \text { for every } n<m
$$

then

$$
\sum_{i=1}^{n} \alpha_{i} \beta_{i}+\partial_{l}(n) C(l)<\left(\sum_{i=1}^{n} \alpha_{i}^{1 / k} \cdot w_{i}\right)^{k} \text { for } n<m
$$


Proof. If $n \leqslant m$, we have

$$
\begin{aligned}
\sum_{i=1}^{n} \alpha_{i} \beta_{i}+\partial_{l}(n) C(l)= & \alpha_{n}\left(\sum_{i=1}^{n} \beta_{i}\right)+\left(\alpha_{n-1}-\alpha_{n}\right)\left(\sum_{i=1}^{n-1} \beta_{i}\right) \\
& +\cdots+\left(\alpha_{1}-\alpha_{2}\right) \beta+\partial_{l}(n) C(l) \\
\leqslant & (\operatorname{by}(5.2)) \\
\leqslant & \alpha_{n} s_{n}^{k}+\left(\alpha_{n-1}-\alpha_{n}\right) s_{n-1}^{k}+\cdots+\left(\alpha_{1}-\alpha_{2}\right) s_{1}^{k}+\partial_{l}(n) C(l) \\
\leqslant & (\text { by }(5.1)) \leqslant\left(\sum_{i=1}^{n} \alpha_{i}^{1 / k} \cdot w_{i}\right)^{k} \cdot \square
\end{aligned}
$$

Using Lemma 5.2 we can prove now

Proposition 5.3. If $p=1 / k, 1<k \in \mathbf{N}$, and $E$ is a positive and contractive complemented sublattice of $d(w, p)$, then there is a sequence of finite pairwise disjoint subsets $\left(\sigma_{n}\right)_{n=1}^{\infty}$ of $\mathbf{N}$ such that $u_{n}=\left(\sum_{i=1}^{\bar{\sigma}_{n}} w_{i}\right)^{-k}\left(\sum_{i \in \sigma_{n}} x_{i}\right)$ for $n \in \mathbf{N}$, constitute a normalized basis of $E, \overline{\bar{\sigma}}_{n}$ being the cardinal number of $\sigma_{n}$.

Proof. By Proposition 4.7, $E$ has a normalised basis $\left(u_{n}\right)_{n=1}^{\infty}$ of positive pairwise disjoint elements of $E$. Let $u_{n}=\sum_{i \in \sigma_{n}} \alpha_{i n} x_{i}$, where $\left(\sigma_{n}\right)_{n=1}^{\infty}$ is a sequence of pairwise disjoint subsets of $\mathbf{N}$ and $\alpha_{i n}>0$ for $i \in \sigma_{n}, n \in \mathbf{N}$.

If $\overline{\bar{\sigma}}_{n}=+\infty$, then we write $\sigma_{n}=\left\{i_{j}: j \in \mathbf{N}\right\}$, and, since $\left(u_{n}\right)_{n=1}^{\infty}$ is normalised, it follows that $\left(\alpha_{i_{j}}\right)_{j=1}^{\infty} \in d(w, p)$. Consequently there exists a permutation of integers $\pi_{n}$ such that $\pi_{n}\left(\underline{\sigma}_{n}\right)=\sigma_{n},\left(\alpha_{n_{n}\left(i_{j}\right) n}\right)_{j=1}^{\infty}$ is a decreasing sequence, and $\pi_{n}(i)=i$ for every $i \notin \sigma_{n}$. If $\overline{\bar{\sigma}}_{n}<\infty$, such a permutation $\pi_{n}$ exists obviously. Since the norm \|\|$_{p, w}$ is invariant under permutations of integers, we can find an isometry $T: d(w, p) \rightarrow d(w, p)$ such that $T\left(u_{m}\right)=t_{m}$, where $t_{m}=\sum_{j=1}^{\infty} \alpha_{\pi_{m}(i) m}$ for every $m \in \mathbf{N}$. Then $Q=T P T^{-1}: d(w, p) \rightarrow \overline{\mathrm{Sp}}\left\{t_{m}: m \in \mathbf{N}\right\}$ is a positive and contractive projection whenever $P$ is a positive and contractive projection onto $E$. Consequently, we may assume that the coefficients $\left(\alpha_{i m}\right)_{i \in \sigma_{m}}$ of $u_{m}$ are decreasingly ordered for every $m \in N$. Since $P$ is a positive projection, we have

$$
P\left(x_{i}\right)=\beta_{i n} u_{n} \quad \text { where } \beta_{i n} \geqslant 0 \text { for every } i \in \sigma_{n}, n \in \mathbf{N},
$$

and moreover

$$
\sum_{i \in \sigma_{n}} \alpha_{i n} \beta_{i n}=1=\left(\sum_{j=1}^{\infty}\left(\alpha_{i j n}\right)^{1 / k} \cdot w_{j}\right)^{k} \quad \text { for every } n \in \mathbf{N} .
$$

By (5.4) and using the fact that $\|P\| \leqslant 1$ we have

$$
\left\|\sum_{n=1}^{\infty}\left(\sum_{i \in \sigma_{n}} \gamma_{i} \beta_{i n}\right) u_{n}\right\|_{p, w} \leqslant \sup _{\pi} \sum_{i=1}^{\infty}\left|\gamma_{\pi(i)}\right|^{1 / k} \cdot w_{i}
$$

for every element $\left(\gamma_{i}\right)_{i=1}^{\infty} \in d(w, p)$. Consequently, taking $\gamma_{i_{1}}=\gamma_{i_{2}}=\cdots=\gamma_{i_{m}}=$ 1 and $\gamma_{j}=0$ for $j \neq i_{1}, \ldots, i_{m}$, where $m \leqslant \overline{\bar{\sigma}}_{n}$, we obtain

$$
\sum_{j=1}^{m} \beta_{i_{j} n}<s_{m}^{k} \text { for } m<\overline{\bar{\sigma}}_{n}, n \in \mathbf{N} \text {. }
$$


If $\overline{\bar{\sigma}}_{n}=\infty$, then, since $\lim _{j} \alpha_{i_{j} n}=0$, there is an integer $l \in \mathrm{N}$ such that $\alpha_{i, n}>\alpha_{i_{++1} n}$ and $\alpha_{i, n}=\alpha_{i_{j+1} n}$ for $j \leqslant l-1$.

By (5.3) we have

$$
\sum_{j=1}^{m} \alpha_{i_{j} n} \beta_{i_{j} n}+C(l) \leqslant\left(\sum_{j=1}^{m} \alpha_{i_{j} n}^{1 / k} \cdot w_{j}\right)^{k} \text { for every } m \geqslant l+1 .
$$

Now, by passing to the limit over $m \rightarrow \infty$ in the previous inequality, we obtain, in view of (5.5).

$$
1=\sum_{j=1}^{\infty} \alpha_{i, n} \beta_{i, n}+C(l) \leqslant\left(\sum_{j=1}^{\infty} \alpha_{i, n}^{1 / k} \cdot w_{j}\right)^{k}=1 .
$$

Since $C(l)>0$, this is a contradiction, hence

$$
\overline{\bar{\sigma}}_{n}<\infty \text { for every } n \in \mathbf{N} \text {. }
$$

Then, reasoning as above, we get that $\alpha_{i n}=\alpha_{j n}$ for every $i, j \in \sigma, n \in \mathbf{N}$, consequently $\alpha_{i n}=\left(\sum_{i=1}^{\overline{\bar{o}}_{n}} w_{i}\right)^{-k}$ for every $i \in \sigma_{n}, n \in \mathbf{N}$.

We study now the existence of positive and contractive projections onto a one dimensional sublattice of $d(w, p), p=1 / k$.

Proposition 5.4. (a) Let $p=1 / k, 1<k \in \mathbf{N}$, and $u=\sum_{i \in \sigma} \alpha_{i} x_{i}$, where $\|u\|_{p, w}$ $=1$ and $\alpha_{i} \geqslant 0$ for every $i \in \sigma$. Then there exists $a$ positive and contractive projection $P: d(w, p) \rightarrow \operatorname{Sp}\{u\}$ if and only if the following conditions are satisfied: $\overline{\bar{\sigma}}=n, \alpha_{i_{1}}=\cdots=\alpha_{i_{n}}=\left(\sum_{i=1}^{n} w_{i}\right)^{-k}$, and there exist the positive numbers $\beta_{1}$ $\geqslant \cdots \geqslant 0$ such that

$$
\sum_{i=1}^{m} \beta_{i} \leqslant\left(\sum_{i=1}^{m} w_{i}\right)^{k}=s_{m}^{k} \text { for } m<n,
$$

and

$$
\sum_{i=1}^{n} \beta_{i}=s_{n}^{k}
$$

Then $P\left(\sum_{i=1}^{\infty} \alpha_{i} x_{i}\right)=\left(\sum_{i \in \sigma} \alpha_{i} \beta_{i}\right) u$ for every $\sum_{i=1}^{\infty} \alpha_{i} x_{i} \in d(w, p)$.

(b) If $u=s_{n}^{-k}\left(\sum_{i=1}^{n} x_{i}\right)$ and $t_{n}=(1 / n) s_{n}^{k} \leqslant t_{m}$ for every $1<m \leqslant n$, then the operator defined by

$$
P\left(\sum_{i=1}^{\infty} \alpha_{i} x_{i}\right)=\frac{1}{n}\left(\sum_{i=1}^{n} \alpha_{i}\right)\left(\sum_{i=1}^{n} x_{i}\right)
$$

is a positive and contractive projection onto $\operatorname{Sp}\{u\}$.

(c) If $w_{1}^{k}<t_{n}$ for every $n>1$ and $u$ is as at the point (a), then there is a positive and contractive projection $P: d(w, p) \rightarrow \operatorname{Sp}\{u\}$ if and only if $\overline{\bar{\sigma}}=1$.

Proof. (a) If $P: d(w, p) \rightarrow \operatorname{Sp}\{u\}$ is a positive and contractive projection then, by Proposition 5.3, $\overline{\bar{\sigma}}=n<\infty$ and $\alpha_{i_{1}}=\cdots=\alpha_{i_{n}}=s_{n}^{-k}$. Denote now by $\left(\beta_{j}\right)_{j=1}^{n}$ the coefficients of $\left(P\left(x_{i}\right)\right)_{j=1}^{n}$ decreasingly ordered. Then

$$
\sum_{j=1}^{m} \beta_{j}=\left\|\left(\sum_{j=1}^{m} \beta_{j}\right) u\right\|_{p, w}^{k}=\left\|P\left(\sum_{j \in H} x_{i j}\right)\right\|_{p, w}^{k}<\left\|\sum_{j \in H} x_{i j}\right\|_{p, w}^{k}=s_{m}^{k},
$$


where $H \subseteq \sigma$ with $\overline{\bar{H}}=m \leqslant n$. If $m=n$, then

$$
\sum_{j=1}^{n} \beta_{j}=\left\|P\left(\sum_{j=1}^{n} x_{i_{j}}\right)\right\|_{p, w}^{k}=\left\|\sum_{j=1}^{n} x_{i_{j}}\right\|_{p, w}^{k}=s_{n}^{k} .
$$

Consequently all the conditions are satisfied and obviously $P\left(\sum_{i=1}^{\infty} \alpha_{i} x_{i}\right)=$ $\left(\sum_{i \in \sigma} \alpha_{i} \beta_{i}\right) u$ for every $\sum_{i=1}^{\infty} \alpha_{i} x_{i} \in d(w, p)$.

Conversely, by (5.3), $\sum_{i=1}^{n} \alpha_{i} \beta_{i} \leqslant\left(\sum_{i=1}^{n} \alpha_{i}^{1 / k} \cdot w_{i}\right)^{k}$ for every $\alpha_{1}>\alpha_{2} \geqslant \cdots \geqslant 0$, and, consequently, $\left|\sum_{i=1}^{n} \alpha_{i} \beta_{i}\right| \leqslant \sup _{\pi}\left(\sum_{i=1}^{n}\left|\alpha_{\pi(i)}\right|^{1 / k} w_{i}\right)^{k}$ for all scalars $\left(\alpha_{i}\right)_{i=1}^{n}$. Thus the operator defined by $P\left(\sum_{i=1}^{\infty} \alpha_{i} x_{i}\right)=\left(\sum_{i \in \sigma} \alpha_{i} \beta_{i}\right) u$ is well defined, $\|P\| \leqslant 1$ and $P \geqslant 0$. Since $P(u)=s_{n}^{-k}\left(\sum_{i=1}^{n} \beta_{i}\right) u=u$ (by (5.10)), it follows moreover that $P$ is a projection.

(b) If $t_{n} \leqslant t_{m}$ for every $m \leqslant n$, then, putting $\beta_{1}=\cdots=\beta_{n}=t_{n}$, the relations (5.9) and (5.10) are verified, consequently $P\left(\sum_{i=1}^{\infty} \alpha_{i} x_{i}\right)=(1 / n)\left(\sum_{i=1}^{n} \alpha_{i}\right)\left(\sum_{i=1}^{n} x_{i}\right)$ is a positive and contractive projection.

(c) If there exists $P: d(w, p) \rightarrow \operatorname{Sp}\{u\}$ a positive and contractive projection and if $\overline{\bar{\sigma}}>1$, then by (a) it follows that $(1 / n) s_{n}^{k}=(1 / n) \sum_{i=1}^{n} \beta_{i} \leqslant \beta_{1} \leqslant w_{1}^{k}$ for $n>1$, which is a contradiction.

We can now state the main result of this section:

TheOREM 5.5. If $p=1 / k, 1<k \in \mathbf{N}$, and $w_{1}^{k}<(1 / n)\left(\sum_{i=1}^{n} w_{i}\right)^{k}$ for every $n>1$, then the positive and contractive complemented sublattices $E$ of $d(w, p)$ coincide with the (closed) order ideals of $d(w, p)$. In particular any positive and contractive complemented sublattice of $d(w, p)$ cannot be linearly homeomorphic to $l_{p}$.

Proof. If $E$ is a closed order ideal of $d(w, p)$, then $E=\overline{\operatorname{Sp}}\left\{x_{i}: i \in A \subset \mathbf{N}\right\}$, hence there is a positive and contractive projection $P: d(w, p) \rightarrow E$. Conversely, if $E$ is a positive and contractive complemented sublattice of $d(w, p)$, by Proposition 5.3, it follows that $E=\overline{\operatorname{Sp}}\left\{u_{n}: n \in \mathbf{N}\right\}$, where $u_{n}=\sum_{i \in \sigma_{n}} \alpha_{i} x_{i}>0$ for $n \in \mathbf{N}$ and $\left(\sigma_{n}\right)_{n=1}^{\infty}$ is a sequence of finite pairwise disjoint subsets of $\mathbf{N}$.

By Proposition 5.4(c) it follows that $\overline{\bar{\sigma}}_{n}=1$ for every $n \in \mathbf{N}$. Consequently $E$ is a closed ideal of $d(w, p)$.

The second assertion is an obvious corollary of the first.

The conditions of Theorem 5.5 are verified for example by the spaces $d(w, p)$, $p=1 / k, 1<k \in \mathbf{N}$, with $w_{i}=1 / i^{\alpha}, 0<\alpha<\frac{1}{2}$. Indeed,

$$
\sum_{i=1}^{n} w_{i} \geqslant \int_{1}^{n+1} \frac{d x}{x^{\alpha}}=\frac{1}{1-\alpha}\left[(1+n)^{1-a}-1\right]>n^{1 / k} \quad \text { for every } n>1 .
$$

$d(w, 1 / k)$, where $1<k \in \mathbf{N}$ and $w_{n}=1 / n^{\alpha}$ for $1>\alpha>1-1 / k$ and $n \in \mathbf{N}$, are examples for spaces $d(w, p)$ for which there is a one dimensional positive and contractive complemented sublattice which is not an order ideal of $d(w, p)$.

More precisely, for a fixed $m \in \mathbf{N}$, there are $n>m$ and a positive and contractive projection onto $\operatorname{Sp}\{u\}$, where $u=\left(\sum_{i=1}^{n} w_{i}\right)^{-1}\left(\sum_{i=1}^{n} x_{i}\right)$. Indeed, using the notations of Lemma 5.4(b) we have

$$
t_{n} \leqslant \frac{1}{n}\left(\int_{0}^{n} \frac{d x}{x^{\alpha}}\right)^{k}=\frac{n^{k(1-\alpha)}}{(1-\alpha)^{k} \cdot n} \leqslant \frac{1}{(1-\alpha)^{k} \cdot n^{1-k+\alpha k}} \quad \text { for every } n \in \mathbf{N}
$$


Consequently, for $0<\varepsilon<\frac{1}{2} \min _{1<i<m} t_{i}$, there is a lowest $n>m$ with $t_{n}<\varepsilon$, hence $t_{n} \leqslant t_{j}$ for every $1 \leqslant j \leqslant n$. We conclude applying Proposition 5.4(b).

Theorem 5.5 suggests the following version of Problem 3.

Problem $3 b$. Let $d(w, p), 0<p<1$. Is there a positive and contractive complemented sublattice of $d(w, p)$ linearly homeomorphic to $l_{p}$ ?

6. The representation of $d(w, 1 / k)$ for $1<k \in \mathbf{N}$. In this section we give a representation of the dual of $d(w, 1 / k), 1<k \in \mathbf{N}$, and also a representation of the Mackey completion of this space. These representations seem to be useful in the study of the $d(w, p)$ 's structure. Remark first that the dual of $d(w, p), 0<p<$ 1 , is

$$
\begin{aligned}
d(w, p)^{\prime}=\left\{\left(b_{i}\right)_{i=1}^{\infty}:\right. & \sum_{i=1}^{\infty}\left|a_{i}\right|\left|b_{\pi(i)}\right|<\infty \text { for every permutation } \pi \\
& \text { of the integers and for every } \left.a+\left(a_{i}\right)_{i=1}^{\infty} \in d(w, p)\right\} .
\end{aligned}
$$

Then we have

Proposition 6.1. Let $d(w, p)$, where $p=1 / k, k>1$. Then

$$
\begin{aligned}
d(w, p)^{\prime} \cap c_{0} & =c_{0} \cap\left\{\left(b_{i}\right)_{i=1}^{\infty}: \sup _{n}\left(\sum_{i=1}^{n} b_{i}^{*}\right) /\left(\sum_{i=1}^{n} w_{i}\right)^{k}<\infty\right\}=E, \\
\|b\|_{p, w}^{\prime} & =\sup _{n}\left(\sum_{i=1}^{n} b_{i}^{*}\right) /\left(\sum_{i=1}^{n} w_{i}\right)^{k},
\end{aligned}
$$

where $b=\left(b_{i}\right)_{i=1}^{\infty} \in c_{0}$ and $b^{*}=\left(b_{i}^{*}\right)_{i=1}^{\infty}$ is a decreasingly rearrangement of $b$.

Proof. Let $b \in d(w, p)^{\prime} \cap c_{0}, t_{n}=\sum_{i=1}^{n} b_{i}^{*}$ and $s_{n}=\sum_{i=1}^{n} w_{i}$. Suppose first that

$$
\sup _{n} \frac{t_{n}}{s_{n}^{k}}=+\infty \text {. }
$$

Since $\lim _{n} s_{n}=+\infty$, it follows that $\lim _{n} t_{n}=+\infty$.

Let $n_{0}=0$ and $s_{n_{0}}=0$. If $m \in \mathbf{N}$ is fixed, then (6.3) implies that

$$
\sup _{n} \frac{t_{n}-t_{m}}{\left(s_{n}-s_{m}\right)^{k}}=+\infty
$$

Choosing $n_{1}$ arbitrarily we can find $n_{2} \in \mathrm{N}, n_{2}>n_{1}$ such that

$$
\begin{aligned}
& s_{n_{2}}-s_{n_{1}} \geqslant s_{n_{1}}-s_{n_{0}} \quad\left(\text { since } \lim _{n} s_{n}=\infty\right), \\
& t_{n_{2}}-t_{n_{1}} \geqslant 2^{2 k-1}\left(s_{n_{2}}-s_{n_{1}}\right)^{k} \quad(\text { by (6.4)). }
\end{aligned}
$$

By induction we get an increasing sequence of integers $\left(n_{j}\right)_{j=1}^{\infty}$ such that

$$
\begin{aligned}
& s_{n_{j+1}}-s_{n_{j}}>s_{n_{j}}-s_{n_{j-1}} \\
& t_{n_{j+1}}-t_{n_{j}} \geqslant(j+1)^{2 k-1}\left(s_{n_{j+1}}-s_{n_{j}}\right)^{k}
\end{aligned}
$$


Now let $n_{j-1}<i \leqslant n_{j}$ and $y_{i}=j^{-2 k}\left(s_{n_{j}}-s_{n_{j-1}}\right)^{-k}$. Then $y=\left(y_{i}\right)_{i=1}^{\infty}$ is a decreasing sequence (by (6.5)) and moreover

$$
\sum_{i=1}^{\infty} y_{i}^{1 / k} \cdot w_{i}=\sum_{j=1}^{\infty} \sum_{i=n_{j-1}+1}^{n_{j}} \frac{w_{i}}{j^{2}\left(s_{n_{j}}-s_{n_{j-1}}\right)}=\sum_{j=1}^{\infty} l / j^{2}<\infty,
$$

that is $y \in d(w, p)$. On the other hand

$$
\sum_{i=1}^{\infty} y_{i} b_{i}^{*}=\sum_{j=1}^{\infty} \sum_{i=n_{j-1}+1}^{n_{j}} \frac{t_{n_{j}}-t_{n_{j-1}}}{j^{2 k}\left(s_{n_{j}}-s_{n_{j-1}}\right)^{k}} \geqslant(\text { by }(6.6)) \geqslant \sum_{j=1}^{\infty} \sum_{i=n_{j-1}+1}^{n_{j}} \frac{1}{j}=+\infty \text {, }
$$

which contradicts the fact that $b \in d(w, p)^{\prime}$. Consequently (6.3) is not true and then

$$
\lambda_{k}=\sup _{n} \frac{t_{n}}{s_{n}^{k}}<+\infty
$$

Hence $d(w, p)^{\prime} \cap c_{0} \in E$.

Now let $b \in E$. If the decreasing sequence $y=\left(y_{i}\right)_{i=1}^{\infty} \in d(w, p)$, then

$$
\begin{aligned}
\sum_{i=1}^{n} y_{i} b_{i}^{*} & =\sum_{i=1}^{n-1} t_{i}\left(y_{i}-y_{i+1}\right)+t_{n} y_{n} \\
& \leqslant(\operatorname{by}(6.7)) \leqslant \lambda_{k}\left[\sum_{i=1}^{n-1} s_{i}^{k}\left(y_{i}-y_{i+1}\right)+s_{n}^{k} y_{n}\right] \\
& \leqslant(\text { by }(5.1)) \leqslant \lambda_{k}\left(\sum_{i=1}^{n} y_{i}^{1 / k} \cdot w_{i}\right)^{k} .
\end{aligned}
$$

Hence $\|b\|_{p, w}^{\prime}=\left\|b^{*}\right\|_{p, w}^{\prime} \leqslant \sup _{n} t_{n} / s_{n}^{k}$ and $b \in d(w, p)^{\prime} \cap c_{0}$. On the other hand, for every $\varepsilon>0$, there is $n \in \mathbf{N}$ with $t_{n} / s_{n}^{k}>\lambda_{k}-\varepsilon$. Let

$$
y_{i}= \begin{cases}s_{n}^{-k} & \text { if } i<n, \\ 0 & \text { if } i>n .\end{cases}
$$

Then $\|y\|_{p, w}=1$ and $\|b\|_{p, w}^{\prime}=\left\|b^{*}\right\|_{p, w}^{\prime}>\sum_{i=1}^{n} y_{i} b_{i}=t_{n} / s_{n}^{k}>\lambda_{k}-\varepsilon$. $\varepsilon$ being arbitrarily small, it follows that $\|b\|_{p, w}^{\prime}=\sup _{n} t_{n} / s_{n}^{k}$, consequently the equalities (6.2) are satisfied.

COROLlaRY 6.2. Let $p=1 / k, k>1$.

(a) If $d(w, p) \notin l_{1}$ then

$$
\begin{aligned}
d(w, p)^{\prime} & =\left\{\left(b_{i}\right)_{i=1}^{\infty} \in c_{0}: \sup _{n}\left(\sum_{i=1}^{n} b_{i}^{*}\right) /\left(\sum_{i=1}^{n} w_{i}\right)^{k}<\infty\right\} \\
& =\left\{\left(b_{i}\right)_{i=1}^{\infty}: \sup _{n}\left(\sum_{i=1}^{n} b_{i}^{*}\right) /\left(\sum_{i=1}^{n} w_{i}\right)^{k}<\infty\right\} .
\end{aligned}
$$

(b) If $d(w, p) \subset l_{1}$, then $d(w, p)^{\prime}=l_{\infty}$.

Proof. The case (b) is clear by Proposition 3.4.

(a) Let $b \in d(w, p)^{\prime}$. If $b \notin c_{0}$, then there are $\varepsilon>0$ and $\left(n_{j}\right)_{j=1}^{\infty}$ such that $\left|b_{n}\right|>\varepsilon$ for every $j \in \mathbf{N}$. Reasoning as in the proof of (3.3) we get a contradiction. Hence 
$d(w, p)^{\prime} \subset c_{0}$, consequently, by (6.2), it follows the first equality. By the last part of the proof of Proposition 6.1 it follows the second equality.

We state now the main result of this section:

TheOREM 6.3. Let $p=1 / k<1$ and $d(w, p) \nsubseteq l_{1}$. If there is a positive decreasing sequence $\left(v_{n}\right)_{n=1}^{\infty} \in c_{0}$ such that

$$
\sum_{i=1}^{n} v_{i} \sim\left(\sum_{i=1}^{n} w_{i}\right)^{k} \text { for every } n \in \mathbf{N}
$$

(i.e. there are constants $A, B>0$ such that $A\left(\sum_{i=1}^{n} v_{i}\right) \leqslant\left(\sum_{i=1}^{n} w_{i}\right)^{k}<B\left(\sum_{i=1}^{n} v_{i}\right)$ for every $n \in \mathbf{N}$ ), then

$$
\widetilde{d(w, p)} \approx d(v, 1)
$$

Proof. Theorem 11 of [4] says that $d(v, 1)^{\prime}=\left\{\left(b_{i}\right)_{i=1}^{\infty}: \sup _{n}\left(\sum_{i=1}^{n} b_{i}^{*}\right) /\left(\sum_{i=1}^{n} v_{i}\right)\right.$ $<\infty\}$. Then by the hypothesis and by Corollary 6.2(a) it follows that

$$
d(w, p)^{\prime}=d(v, 1)^{\prime} .
$$

It is easy to see that the canonical basis $\left(x_{n}\right)_{n=1}^{\infty}$ of $d(w, p)$ is a symmetric basis of $\widetilde{d(w, p)}$ too. Then, denoting by $\widetilde{d(w, p)^{x}}$ the Köthe dual of $\widetilde{d(w, p)}$, that is the space $\left\{\left(b_{i}\right)_{i=1}^{\infty}: \sum_{i=1}^{\infty}\left|a_{i} b_{i}\right|<+\infty\right.$ for every $\left.a=\left(a_{i}\right)_{i=1}^{\infty} \in \widehat{d(w, p)}\right\}$, it follows that $\widetilde{d(w, p)^{x}}$ $=d(w, p)^{x}=d(w, p)^{\prime}=\widetilde{d(w, p)^{\prime}}$. Consequently $\widetilde{d(w, p)^{x x}}=\widetilde{d(w, p)^{\prime x}}=($ by (6.10)) $=d(v, 1)^{\prime x}=($ by Corollary of Theorem 10 of $[4])=d(v, 1)^{x x}$. We shall show that $d(v, 1)^{x x}=d(v, 1)$.

A Köthe result (see $\$ 30$, p. 5 of [6]) says that for a Banach sequence space $E$, $E^{x x}=E$ if and only if $E$ is weakly sequentially complete. Hence, it suffices to show that $d(w, 1)$ is weakly sequentially complete. By Theorem $1 . c .10$ of [8] it follows that we must show that $c_{0} \downarrow d(v, 1)$. If $c_{0} \subset d(v, 1)$, Corollary 17 of [3] implies that $c_{0}$ contains a complemented subspace linearly homeomorphic to $l_{1}$, which is a contradiction. Hence $\widetilde{d(w, p)^{x x}}=d(v, 1)^{x x}=d(v, 1)$, consequently $\widetilde{d(w, p)} \subset d(v, 1) \subset \widetilde{d(w, p)^{x x}}$. By Proposition 3.3 it follows that every order interval $[0, x] \subset \overline{d(w, p)}$ is compact. On the other hand Theorem II. 5.10 of [11] shows us that in a Banach lattice $E$ the following assertions are equivalent:

(1) The norm of $E$ is order continuous (i.e. if $\left(y_{\alpha}\right)_{\alpha \in A}$ is a downward directed set in $E$ with $\inf _{\alpha} y_{\alpha}=0$, then $\lim _{\alpha}\left\|y_{\alpha}\right\|=0$ ).

(2) Every order interval $[0, x] \subset E$ is $\sigma\left(E, E^{\prime}\right)$-compact.

(3) $E$ is an order ideal in $E^{\prime \prime}$.

Finally, a theorem of Ando (see Proposition IV.11.1 of [11]) says that a Banach lattice $E$ has an order continuous norm if and only if every closed order ideal of $E$ is the range of a positive projection from $E$.

Consequently $\widetilde{d(w, p)}$ is a complemented subspace, with a symmetric basis $\left(y_{n}\right)_{n}$, of the space $\widetilde{d(w, p)^{\prime \prime}}=\widetilde{d(w, p)^{x x}}=d(v, 1)$. Since $\widetilde{d(w, p)} \approx l_{1}$, by the Corollary 14 of [3] it follows that $\overline{d(w, p)} \approx d(v, 1)$.

REMARK 6.4. (1) $d(w, 1 / k)$ with $k>1$ and $w_{n}=1 / n^{\alpha}$, where $(k-1) / k<\alpha<$ 1 , are examples of the spaces $d(w, 1 / k)$ which verify the conditions of Theorem 6.3. Indeed, if $\alpha=1$, we take in Proposition 3.7, $\gamma=0, b=1$ and $n_{j}=j$ for every $j \in \mathbf{N}$. Hence $d(w, 1 / k) \nsubseteq l_{1}$. 
If $(k-1) / k<\alpha<1$, let $0<\gamma<1 /(k-1), n_{j}=j^{1 / \alpha}$ for every $j \in \mathbf{N}$, and $b=\sup _{j}\left[(j+1)^{1 / \alpha}-j^{1 / \alpha}\right] / j^{\gamma}<+\infty$. Then, by Proposition 3.7, it follows that $d(w, 1 / k) \notin l_{1}$. Suppose now again that $\alpha=1$ and put

$$
v_{i}= \begin{cases}1 & \text { if } i \leqslant\left[e^{k-1}\right]+1, \\ \frac{(\log i)}{i}^{k-1} & \text { if } i>\left[e^{k-1}\right]+1\end{cases}
$$

where $\left[e^{k-1}\right]$ is the entire part of $e^{k-1}$. Then

$$
\sum_{i=1}^{n} v_{i} \sim \int_{3}^{n} \frac{(\log x)^{k-1}}{x} d x \sim(\log n)^{k} \sim\left(\sum_{i=1}^{n} w_{i}\right)^{k} \text { for every } n .
$$

Moreover $\lim _{n} v_{n}=0$ and $v_{n} \geqslant v_{n+1}$ for every $n \in \mathbf{N}$.

If $(k-1) / k<\alpha<1$, then we put $v_{n}=1 / n^{\beta}$, where $\beta=k(\alpha-(k-1) / k)<$ 1 for every $n \in \mathbf{N}$. Clearly $\lim _{n} v_{n}=0$ and $\left(v_{n}\right)_{n=1}^{\infty}$ is a decreasing sequence. Moreover

$$
\sum_{i=1}^{n} v_{i} \sim \int_{1}^{n} \frac{d x}{x^{\beta}} \sim n^{1-\beta} \sim n^{k(1-\alpha)} \sim\left(\sum_{i=1}^{n} w_{i}\right)^{k} \quad \text { for every } n \in \mathbf{N}
$$

(2) We do not know if the condition (6.8) is superfluous.

Let us mention the following problem:

Problem 4. Let $p=q / k, 1<q<k$. Is there a positive decreasing sequence $\left(v_{i}\right)_{i=1}^{\infty} \in c_{0} \backslash l_{1}$ such that $\overline{d(w, p)} \approx d(v, q)$ ?

More generally:

Problem 4a. Let $0<p<1, p \neq 1 / k$ for any $k \in \mathbf{N}$. Is $\widetilde{d(w, p)}$ reflexive?

Remark that a positive answer to Problem $4 \mathrm{a}$ implies a positive answer to Problem 3 for $p \neq 1 / k$.

\section{REFERENCES}

1. Z. Altshuler, P. G. Cassaza and B. L. Lin, On symmetric basic sequences in Lorentz sequence spaces, Israel J. Math. 15 (1973), 140-155.

2. G. Bennett, An extension of the Riesz-Thorin theorem, Lecture Notes in Math., vol. 604, Springer-Verlag, Berlin and New York, 1977.

3. P. G. Cassaza and B. L. Lin, On symmetric basic sequences in Lorentz sequence spaces. II, Israel J. Math. 17 (1974), 191-218.

4. D. J. H. Garling, On symmetric sequence spaces, Proc. London Math. Soc. 16 (1966), 85-106.

5. N. J. Kalton, Orlicz sequence spaces without local convexity, Proc. Cambridge Philos. Soc. 81 (1977), 253-277.

6. G. Köthe, Topological vector spaces. I, Springer-Verlag, Berlin and New York, 1969.

7. J. Lindenstrauss and A. PeXczyński, Absolutely summing operators in $\mathcal{E}_{p}$-spaces and their applications, Studia Math. 29 (1968), 275-326.

8. J. Lindenstrauss and L. Tzafriri, Classical Banach spaces I. Sequence spaces, Springer-Verlag, Berlin and New York, 1977.

9. B. Maurey, Théorèmes de factorisation pour les opérateurs linéaires à valeurs dans les espaces $L^{p}$, Astérisque 11 (1974), 1-163.

10. S. Rolewicz, Metric linear spaces, PWN, Warszawa, 1972. 
11. H. H. Schaefer, Banach lattices and positive operators, Springer-Verlag, Berlin and New York, 1974.

12. W. J. Stiles On properties of subspaces of $l_{p}, 0<p<1$, Trans. Amer. Math. Soc. 149 (1970), 405-415.

13. B. Walsh, On characterizing Köthe sequence spaces as vector lattices, Math. Ann. 175 (1968), 253-256.

Department of MAthematics, INCREST, Bucharest 77538, Romania

Current address: Mathematisches Institut, Universität des Saarlandes, Saarbrücken 6600, West Germany 